\title{
A spectral-based numerical method for Kolmogorov equations in Hilbert spaces.
}

\author{
Francisco Delgado-Vences* and Franco Flandolit
}

October 11, 2018

\begin{abstract}
We propose a numerical solution for the solution of the Fokker-Planck-Kolmogorov (FPK) equations associated with stochastic partial differential equations in Hilbert spaces. The method is based on the spectral decomposition of the Ornstein-Uhlenbeck semigroup associated to the Kolmogorov equation. This allows us to write the solution of the Kolmogorov equation as a deterministic version of the Wiener-Chaos Expansion. By using this expansion we reformulate the Kolmogorov equation as a infinite system of ordinary differential equations, and by truncation it we set a linear finite system of differential equations. The solution of such system allow us to build an approximation to the solution of the Kolmogorov equations. We test the numerical method with the Kolmogorov equations associated with a stochastic diffusion equation, a Fisher-KPP stochastic equation and a stochastic Burgers Eq. in dimension 1.
\end{abstract}

\section{Introduction}

Stochastic Partial Differential Equations (SPDE's) are important tools in modeling complex phenomena, they arise in many fields of knowledge like Physics, Biology, Economy, Finance, etc.. Develop efficient numerical methods for simulating SPDE's is very importan but also very difficult and challenging.

There exists in literature several approachs in order to solve numerically a SPDE. Among them there exists Monte Carlo simulations, Karhunen-Loeve Expansion, Wiener Chaos expansion, stochastic Taylor approximations for SPDE's, etc. In order to solve numerically an SPDE one can apply one of this methods. Here we will mention some of them but our list reference is far away to be exhaustive.

The Monte Carlo (MC) simulations for SPDE's have been explored intensively in the last 20 years ([28], [21] ). The basis idea of MC is to sample the randomness in the SPDE's and solve the stochastic equations realization by realization, this is because for each given realization of the randomness, the SPDE's becomes deterministic and can be solved by usual deterministic numerical methods. the disadvatage is that many of that "samples" are required for suffcient accuracy, causing suboptimal efficiency even if optimal algebraic solvers are used; to overcome this issue Giles ([13], [14]) has introduce a modificacion of $\mathrm{MC}$ for the numerical solution of Itô stochastic ordinary differential equations, following basic ideas in earlier work by S. Heinrich [16] on numerical quadrature. This method is the so-called Multilevel Monte Carlo (MLMC).

The main idea of MLMC methods is to apply the MC method for a nested sequence of stepsizes while balancing the number of samples according to the stepsize. MLMC allows to significantly speed up to classical MC methods thanks to this hierarchical sampling; however this method still can have limitations for SPDE's.

Other approach is use spectral methods, in particular use the Karhunen-Loeve expansion (KLE) and the Wiener Chaos expansions for solving SPDE's. For the former one can study the theory developed

\footnotetext{
*Istituto Nazionale di Geofísica e Vulcanología, Pisa, Italy. Email addresses: francisco.delgado@ingv.it, delgadovences@gmail.com

${ }^{\dagger}$ Dipartimento di Matematica, Universitá di Pisa, Largo Bruno Pontecorvo 5, 56127 Pisa, Italy. Email address: flandoli@dma.unipi.it.
} 


\section{INTRODUCTION}

in [22]; in this work they proposed several methods to solve SPDE's and this methods are latter applied to solve the stochastic Navier-Stokes equations. Nevertheless, this method can have limitations since in this approach the source of randomness is usually represented by a fixed number of random variables and if we consider, for instance, stochastic equations arising in fluid dynamics with a random forcing white in time which has a divergent Karhunen-Loeve expansion, then it is not possible to apply the $\mathrm{KLE}$ to this kind of equations.

Hou et al [17] propose a numerical method based on Wiener Chaos expansion and apply it to solve the stochastic Burgers and Navier-Stokes equations driven by Brownian motion. They consider an SPDE with Brownian motion forcing and since a Brownian motion can be expand as a linear combination of independent Gaussian random variables, then they expand the solution of theSPDE's as a Fourier-Hermite series of those Gaussian random variables, this is a version of the Cameron-Martin decomposition.

There is another approach that involves stochastic Taylor approximations for stochastic partial differential equations (see [20]). This Taylor expansions are based on an iterated application of the Itô formula. However, For the solutions of stochastic partial differential equations in Hilbert (or Banach spaces) there is no way to define directly the Itô formula. Nevertheless, it can be constructed by taking advantage of the mild form representation of the solutions.

The Fokker-Planck-Kolmogorov (FPK) equation is a partial differential equation that describes the time evolution of the probability density function of the velocity of a particle under the influence of drag forces and random forces, it is a kind of continuity equation for densities. Citing [9] "parabolic equations on Hilbert spaces appear in mathematical physics to model systems with infinitely many degrees of freedom. Typical examples are provided by spin configurations in statistical mechanics and by crystals in solid state theory. Infinite-dimensional parabolic equations provide an analytic description of infinite dimensional diffusion processes in such branches of applied mathematics as population biology, fluid dynamics, and mathematical finance.". This kind of equations have been deeply studied in the last years, see for instance [2], [11], [7] and the references therein.

Numerical methods for FPK equations associated with SPDEs have been studied, up to our knowledge, just in a few articles, here we will mention just one. Schwab and Suli [29] have formulated a space-time variational method to approximate solution of Kolmogorov-type equations in infinite dimensions. They consider an infinite-dimensional Hilbert space $\mathcal{H}$, a Gaussian measure $\mu$ with trace class covariance operator $Q$ on $\mathcal{H}$ and the space $L^{2}(H, \mu)$ of functions on $\mathcal{H}$ which are square-integrable with respect to the measure $\mu$. They showed the well-posedness of Fokker-Plank equations and Ornstein-Uhlenbeck equations on $L^{2}(H, \mu)$. Moreover, they constructed sequences of finite-dimensional approximations that attain the best possible convergence rates afforded by best $N$-terms approximations of the solution. They used an spectral method based on Wiener-Hermite polynomial chaos expansions in terms of a sequence of independent Gaussian random variables on $\mathcal{H}$ and a Wavelet type Riesz basis with respect to the time variable. The use of the spectral basis of Wiener-Hermite polynomial chaos allow them to avoid meshing the infinite-dimensional "domain" $\mathcal{H}$ of solutions of the Kolmogorov-type equations. However they do not present numerical examples and the questions about the feasibility of their method are open.

In this paper, we introduce a novel numerical method that can have some similitude with the one proposed by Schwab and Suli but also have substantial differences. Indeed, our method is also based on spectral methods for the variable on $\mathcal{H}$, but we use a deterministic version of the Wiener-Chaos Expansion on the infinite-dimensional "domain" $\mathcal{H}$ instead the classical Wiener-Chaos Expansion with the use of a sequence of Gaussian random variables, this allow allow us to avoid meshing the space $\mathcal{H}$ but we also avoid the so-called curse of dimensionality: the associated computational cost grows exponentially as a function of the number of random variables defining the underlying probability space of the problem (see [12] for instance).

The second difference is with respect to the time variable, where, instead using Wavelet type Riesz basis we set up a finite system of coupled ordinary differential equations and by solving it we fix the coefficients as a time functions. we have applied the method to three SPDE's: a stochastic diffusion, a stochastic FisherKPP equation and a stochastic burgers equation and the results show that the behaviour of the 
method is good. However, since the method is analogue to the classical deterministic spectral method thus it can be extended to improve its performance. This is the subject of a future research.

This paper is organized as follows. In section 2 we review the Fokker-Plank-Kolmogorov equation associated with SPDE's in a separable Hilbert space. In section 3 we study the spectral decomposition of the Ornstein-Uhlenbeck semigroup associated to the Kolmogorov equation which will be used to do the numerical approximation to the solution of the FPK equation, this is done in section 4 In section 5 we prove a theorem on the well posedness and convergence of the numerical aproximation. Results on the application of the proposed method are presented in section 6 where we have applied the method to a linear stochastic diffusion equation, a Fisher-KPP stochastic equation and a stochastic Burgers Eq. in dimension 1.

\section{Fokker-Plank-Kolmogorov equation}

In a separable infinite-dimensional Hilbert space $\mathcal{H}$ with inner product $\langle,\rangle_{\mathcal{H}}$ we define a Gaussian measure $\mu$ with mean zero and nuclear covariance operator $\Lambda$ with $\operatorname{Tr}(\Lambda)<+\infty$.

We focus on the stochastic differential equation in $\mathcal{H}$

$$
d X_{t}=A X_{t} d t+B\left(X_{t}\right) d t+\sqrt{Q} d W_{t},
$$

where the operator $A: \mathcal{D}(A) \subset \mathcal{H} \rightarrow \mathcal{H}$ is the infinitesimal generator of a strongly continuous semigroup $e^{t A}$ in $\mathcal{H}, Q$ is a bounded operator from another Hilbert space $\mathcal{U}$ to $\mathcal{H}$ and $B: \mathcal{D}(B) \subset \mathcal{H} \rightarrow \mathcal{H}$ is a nonlinear mapping.

The equation (2.1) can be associated to a Kolmogorov equation in the next way, we define

$$
u(t, x)=\mathbb{E}\left[u_{0}\left(X_{t}^{x}\right)\right],
$$

where $u_{0}: \mathcal{H} \rightarrow \mathbb{R}$ and $X_{t}^{x}$ is the solution to (2.1) with initial conditions $X_{0}=x$ where $x \in \mathcal{H}$. Then $u$ satisfies the Kolmogorov equation

$$
\frac{\partial u}{\partial t}=\frac{1}{2} \operatorname{Tr}\left(Q D^{2} u\right)+\langle A x, D u\rangle_{\mathcal{H}}+\langle B(x), D u\rangle_{\mathcal{H}}, \quad x \in D(A) .
$$

Several authors have proved results on existence and uniqueness of the solution of the Kolmogorov equations, see for instance Da Prato [7] for a survey, Da Prato-Debussche [8] for the Burgers equation, Barbu-Da Prato [1] for the 2D Navier-Stokes stochastic flow in a channel.

\section{On the Ornstein-Uhlenbeck semigroup}

Following [6], in $\mathcal{H}$ we define a Gaussian measure $\mu$ with mean zero and nuclear covariance operator $\Lambda$ with $\operatorname{Tr}(\Lambda)<+\infty$ and since $\Lambda: \mathcal{H} \mapsto \mathcal{H}$ is a positive definite, self-adjoint operator then its square-root operator $\Lambda^{1 / 2}$ is a positive definite, self-adjoint Hilbert-Schmidt operator on $\mathcal{H}$.

Define the inner product

$$
\langle g, h\rangle_{0}:=\left\langle\Lambda^{-1 / 2} g, \Lambda^{-1 / 2} h\right\rangle_{\mathcal{H}}, \quad \text { for } \quad g, h \in \Lambda^{1 / 2} \mathcal{H} .
$$

Let $\mathcal{H}_{0}$ denote the Hilbert subspace of $\mathcal{H}$, which is the completion of $\Lambda^{1 / 2} \mathcal{H}$ with respect to the norm $\|g\|_{0}:=\langle g, g\rangle_{0}^{1 / 2}$. Then $\mathcal{H}_{0}$ is dense in $\mathcal{H}$ and the inclusion map $i: \mathcal{H}_{0} \hookrightarrow \mathcal{H}$ is compact. The triple $\left(i, \mathcal{H}_{0}, \mathcal{H}\right)$ forms an abstract Wiener space.

Let $\mathbb{H}=L^{2}(\mathcal{H}, \mu)$ denote the Hilbert space of Borel measurable functionals on the probability space with inner product

$$
\langle\Phi, \Psi\rangle_{\mathbb{H}}:=\int_{\mathcal{H}} \Phi(v) \Psi(v) \mu(d v), \quad \text { for } \quad \Phi, \Psi \in \mathbb{H},
$$

and norm $\|\Phi\|_{\mathbb{H}}:=\langle\Phi, \Phi\rangle_{\mathbb{H}}^{1 / 2}$. In $\mathbb{H}$ we choose a basis system $\left\{\varphi_{k}\right\}$ such that $\varphi_{k} \in \mathcal{H}$. 
A functional $\Phi: \mathcal{H} \mapsto \mathbb{R}$, is said to be a smooth simple functional (or a cylinder functional) if there exists a $C^{\infty}$-function $\phi$ on $\mathbb{R}^{n}$ and $n$-continuous linear functional $l_{1}, \ldots, l_{n}$ on $\mathcal{H}$ such that for $h \in \mathcal{H}$

$$
\Phi(h)=\phi\left(h_{1}, \ldots, h_{n}\right) \quad \text { where } \quad h_{i}=l_{i}(h), \quad i=1, \ldots, n .
$$

The set of all such functionals will be denoted by $\mathcal{S}(\mathbb{H})$.

Denote by $P_{k}(x)$ the Hermite polynomial of degree $k$ taking values in $\mathbb{R}$. Then, $P_{k}(x)$ is given by the following formula

$$
P_{k}(x)=\frac{(-1)^{k}}{(k !)^{1 / 2}} e^{\frac{x^{2}}{2}} \frac{d^{k}}{d x^{k}} e^{-\frac{x^{2}}{2}}
$$

with $P_{0}=1$. It is well-known that $\left\{P_{k}(\cdot)\right\}_{k \in \mathbb{N}}$ is a complete orthonormal system for $L^{2}\left(\mathbb{R}, \mu_{1}(d x)\right)$ with $\mu_{1}(d x)=\frac{1}{\sqrt{2 \pi}} e^{-\frac{x^{2}}{2}} d x$.

Define the set of infinite multi-index as

$$
\mathcal{J}=\left\{\boldsymbol{\alpha}=\left(\alpha_{i}, i \geq 1\right) \quad\left|\quad \alpha_{i} \in \mathbb{N} \cup\{0\}, \quad\right| \boldsymbol{\alpha} \mid:=\sum_{i=1}^{\infty} \alpha_{i}<+\infty\right\}
$$

For $\boldsymbol{n} \in \mathcal{J}$ define the Hermite polynomial functionals on $\mathcal{H}$ by

$$
H_{\boldsymbol{n}}(h)=\prod_{i=1}^{\infty} P_{n_{i}}\left(l_{i}(h)\right), \quad h \in \mathcal{H}_{0}, \quad \boldsymbol{n} \in \mathcal{J}
$$

and where

$$
l_{i}(h)=\left\langle h, \Lambda^{-1 / 2} \varphi_{i}\right\rangle_{\mathcal{H}}, \quad i=1,2, \ldots
$$

where $P_{n}(\xi)$ is the usual Hermite polynomial for $\xi \in \mathbb{R}$ and $n \in \mathbb{N}$.

Remark 3.1. Notice that $l_{i}(h)$ is defined only for $h \in \mathcal{H}_{0}$. However, regarding $h$ as a $\mu$-random variable in $\mathcal{H}$, we have $\mathbb{E}\left(l_{i}(h)\right)=\left\|\varphi_{i}\right\|^{2}=1$ and then $l_{k}(h)$ can be defined $\mu$-a.e. $h \in \mathcal{H}$, similar to defining a stochastic integral.

It is possible to identify the Hermite polynomial functionals defined in (3.1), for $h \in \mathcal{H}_{0}$, as a deterministic version of the Wick polynomials defined on the canonical Wiener space.(for further details see [19] for instance).

We have the following result (See Theorems 9.1.5 and 9.1.7 in Da Prato-Zabczyk [9] or Lemma 3.1 in chapter 9 from Chow [6]).

Lemma 3.2. For $h \in \mathcal{H}$ let $l_{i}(h)=\left\langle h, \Lambda^{-1 / 2} \varphi_{i}\right\rangle_{\mathcal{H}}, i=1,2, \ldots$ Then the set $\left\{H_{\boldsymbol{n}}\right\}$ of all Hermite polynomials on $\mathcal{H}$ forms a complete orthonormal system for $\mathbb{H}$. Hence the set of all functionals are dense in $\mathbb{H}$. Moreover, we have the direct sum decomposition:

$$
\mathbb{H}=\bigoplus_{j=0}^{\infty} K_{j}
$$

where $K_{j}$ is the subspace of $\mathbb{H}$ spanned by $\left\{H_{\boldsymbol{n}}:|\boldsymbol{n}|=j\right\}$.

\section{Spectral decomposition of the Ornstein-Uhlenbeck semigroup}

Consider the linear stochastic equation

$$
\begin{aligned}
d u_{t} & =A u_{t} d t+d W_{t}, \\
u_{0} & =h \in \mathcal{H} .
\end{aligned}
$$

Here, as before $A: \mathcal{D}(A) \subset \mathcal{H} \rightarrow \mathcal{H}$ is the infinitesimal generator of a strongly continuous semigroup $e^{t A}$ in $\mathcal{H} . W_{t}$ is a $Q$-Wiener process in $\mathcal{H}$.

Chow in [6, Lemma 9.4.1] has shown the following result. 
Lemma 3.3. Suppose that $A$ and $Q$ satisfy the following:

1. $A: \mathcal{D}(A) \subset \mathcal{H} \rightarrow \mathcal{H}$ is self-adjoint and there is $\beta>0$ such that

$$
\langle A v, v\rangle_{\mathcal{H}} \leq-\beta\|v\|_{\mathcal{H}} \quad \forall v \in \mathcal{H} .
$$

2. A commutes with $Q$ in $\mathcal{D}(A) \subset \mathcal{H}$.

Then (3.2) has a unique invariant measure $\mu$ which is a Gaussian measure on $\mathcal{H}$ with zero mean and covariance operator $\Lambda=\frac{1}{2} Q(-A)^{-1}=\frac{1}{2}(-A)^{-1} Q$.

We define the operator

$$
\mathcal{A}_{0} u=\frac{1}{2} \operatorname{Tr}\left(Q D^{2} u\right)+\langle A x, D u\rangle_{\mathcal{H}}, \quad x \in \mathcal{H}
$$

and suppose that $-A$ and $Q$ have the same eigenfunctions $e_{k}$ with eigenvalues $\lambda_{k}$ y $\rho_{k}$ respectively. Then the operator $\mathcal{A}_{0}$ satisfies the following result.

Lemma 3.4. Let $H_{\boldsymbol{n}}(h)$ be a Hermite polynomial functional given by (3.1). Then the following holds

$$
\mathcal{A}_{0} H_{\boldsymbol{n}}(h)=-\lambda_{\boldsymbol{n}} H_{\boldsymbol{n}}(h),
$$

for any $\boldsymbol{n} \in \mathcal{J}$ and $h \in \mathcal{H}$ and where

$$
\lambda_{n}:=\sum_{k=1}^{\infty} n_{k} \lambda_{k}
$$

The proof can be found in [6, Lemma 9.4.3] or [4].

Using lemmas 3.4 and 3.2 , $\left(\left\{H_{\boldsymbol{n}}\right\}\right.$ forms a complete orthonormal system for $\left.L^{2}(\mathcal{H}, \mu)\right)$ we can write

$$
u(t, x)=\sum_{\boldsymbol{n} \in \mathcal{J}} u_{\boldsymbol{n}}(t) H_{\boldsymbol{n}}(x), \quad x \in \mathcal{H}, \quad t \in[0, T],
$$

where $u_{\boldsymbol{n}}:[0, T] \mapsto \mathbb{R}$ and $H_{\boldsymbol{n}}(x)$ are the Hermite functionals.

Remark 3.5. The decomposition given in (3.5) is a deterministic version to the Wiener Chaos expansion (WCe), also known as a Fourier-Hermite series. The WCe has been used to prove several results in stochastic analysis and also it has been applied to solve numerically stochastic partial differential equations (see for instance Lototsky and Rozovskii [25], Lototsky [24], Hou et. al. [17]).

Notice that the Kolmogorov equation can be writen as

$$
\begin{aligned}
\frac{\partial u}{\partial t} & =\frac{1}{2} \operatorname{Tr}\left(Q D^{2} u\right)+\langle A x, D u\rangle_{\mathcal{H}}+\langle B(x), D u\rangle_{\mathcal{H}} \\
& =\mathcal{A}_{0} u+\langle B(x), D u\rangle_{\mathcal{H}} .
\end{aligned}
$$

Using (3.5), we calculate

$$
\begin{aligned}
\frac{\partial u}{\partial t} & =\sum_{\boldsymbol{n} \in \mathcal{J}} \dot{u}_{\boldsymbol{n}}(t) H_{\boldsymbol{n}}(x) \\
\mathcal{A}_{0} u & =\mathcal{A}_{0} \sum_{\boldsymbol{n} \in \mathcal{J}} u_{\boldsymbol{n}}(t) H_{\boldsymbol{n}}(x)=\sum_{\boldsymbol{n} \in \mathcal{J}} u_{\boldsymbol{n}}(t) \mathcal{A}_{0} H_{\boldsymbol{n}}(x) \\
& =-\sum_{\boldsymbol{n} \in \mathcal{J}} u_{\boldsymbol{n}}(t) \lambda_{\boldsymbol{n}} H_{\boldsymbol{n}}(x)
\end{aligned}
$$

Where in the last equality we have used the Lemma 3.4 
For the last term in (3.6) we have

$$
\begin{aligned}
\langle B(x), D u\rangle_{\mathcal{H}} & =\left\langle B(x), D_{x} \sum_{\boldsymbol{n} \in \mathcal{J}} u_{\boldsymbol{n}}(t) H_{\boldsymbol{n}}(x)\right\rangle_{\mathcal{H}} \\
& =\sum_{\boldsymbol{n} \in \mathcal{J}} u_{\boldsymbol{n}}(t)\left\langle B(x), D_{x} H_{\boldsymbol{n}}(x)\right\rangle_{\mathcal{H}},
\end{aligned}
$$

where $D_{x}$ is the Fréchet derivative.

Therefore the Kolmogorov equation becomes

$$
\sum_{\boldsymbol{n} \in \mathcal{J}} \dot{u}_{\boldsymbol{n}}(t) H_{\boldsymbol{n}}(x)=-\sum_{\boldsymbol{n} \in \mathcal{J}} u_{\boldsymbol{n}}(t) \lambda_{\boldsymbol{n}} H_{\boldsymbol{n}}(x)+\sum_{\boldsymbol{n} \in \mathcal{J}} u_{\boldsymbol{n}}(t)\left\langle B(x), D_{x} H_{\boldsymbol{n}}(x)\right\rangle_{\mathcal{H}}
$$

Multiplying by $H_{\boldsymbol{m}}(x), \boldsymbol{m} \in \mathcal{J}$ and integrating in $\mathcal{H}$ w.r.t $\mu(d x)$ we have

$$
\begin{aligned}
\sum_{\boldsymbol{n} \in \mathcal{J}} \dot{u}_{\boldsymbol{n}}(t) \int_{\mathcal{H}} H_{\boldsymbol{m}}(x) H_{\boldsymbol{n}}(x) \mu(d x) & =-\sum_{\boldsymbol{n} \in \mathcal{J}} u_{\boldsymbol{n}}(t) \lambda_{\boldsymbol{n}} \int_{\mathcal{H}} H_{\boldsymbol{m}}(x) H_{\boldsymbol{n}}(x) \mu(d x) \\
& +\sum_{\boldsymbol{n} \in \mathcal{J}} u_{\boldsymbol{n}}(t) \int_{\mathcal{H}} H_{\boldsymbol{m}}(x)\left\langle B(x), D_{x} H_{\boldsymbol{n}}(x)\right\rangle_{\mathcal{H}} \mu(d x)
\end{aligned}
$$

From this, and using the orthogonality of the system $\left\{H_{\boldsymbol{m}}(x)\right\}$ we get the infinite system of coupled ordinary differential equations

$$
\dot{u}_{\boldsymbol{m}}(t)=-u_{\boldsymbol{m}}(t) \lambda_{\boldsymbol{m}}+\sum_{\boldsymbol{n} \in \mathcal{J}} u_{\boldsymbol{n}}(t) C_{\boldsymbol{n}, \boldsymbol{m}}, \quad \boldsymbol{n}, \boldsymbol{m} \in \mathcal{J}
$$

where $C_{n, m}$ is given by

$$
C_{\boldsymbol{n}, \boldsymbol{m}}:=\int_{\mathcal{H}}\left\langle B(x), D_{x} H_{\boldsymbol{n}}(x)\right\rangle_{\mathcal{H}} H_{\boldsymbol{m}}(x) \mu(d x) .
$$

We focus on $C_{\boldsymbol{n}, \boldsymbol{m}}$. Since $H_{\boldsymbol{n}}(x)=\prod_{i=1}^{\infty} P_{n_{i}}\left(\left\langle x, \Lambda^{-\frac{1}{2}} e_{i}\right\rangle_{\mathcal{H}}\right)$ we get

$$
D_{x} H_{\boldsymbol{n}}(x)=\sum_{k=1}^{\infty} \prod_{\substack{i=1 \\ i \neq k}}^{\infty} P_{n_{i}}\left(\left\langle x, \Lambda^{-\frac{1}{2}} e_{i}\right\rangle_{\mathcal{H}}\right) P_{n_{k}}^{\prime}\left(\left\langle x, \Lambda^{-\frac{1}{2}} e_{k}\right\rangle_{\mathcal{H}}\right) \Lambda^{-\frac{1}{2}} e_{k},
$$

then

$$
\left\langle B(x), D_{x} H_{\boldsymbol{n}}(x)\right\rangle_{\mathcal{H}}=\sum_{k=1}^{\infty}\left\langle B(x), \Lambda^{-\frac{1}{2}} e_{k}\right\rangle_{\mathcal{H}} \prod_{\substack{i=1 \\ i \neq k}}^{\infty} P_{n_{i}}\left(\left\langle x, \Lambda^{-\frac{1}{2}} e_{i}\right\rangle_{\mathcal{H}}\right) P_{n_{k}}^{\prime}\left(\left\langle x, \Lambda^{-\frac{1}{2}} e_{k}\right\rangle_{\mathcal{H}}\right)
$$

Thus,

$$
C_{\boldsymbol{n}, \boldsymbol{m}}=\int_{\mathcal{H}} \sum_{k=1}^{\infty}\left\langle B(x), \Lambda^{-\frac{1}{2}} e_{k}\right\rangle_{\mathcal{H}} \prod_{\substack{i=1 \\ i \neq k}}^{\infty} P_{n_{i}}\left(\left\langle x, \Lambda^{-\frac{1}{2}} e_{i}\right\rangle_{\mathcal{H}}\right) P_{n_{k}}^{\prime}\left(\left\langle x, \Lambda^{-\frac{1}{2}} e_{k}\right\rangle_{\mathcal{H}}\right) H_{\boldsymbol{m}}(x) \mu(d x) .
$$

\section{A technical result}

The following result is important for the numerical simulation since it will allow us to use the evaluation functional on the Hilbert space $\mathcal{H}$.

Lemma 3.6. i) The Gaussian measure $\mu$ on $\mathcal{H}=L^{2}(0,1)$ with covariance $\Lambda=\frac{1}{2}(-A)^{-1}$ is supported on $C([0,1])$.

ii) Let $\xi_{0} \in[0,1]$ be given. Let $u_{0}: C([0,1]) \rightarrow \mathbb{R}$ be defined as $u_{0}(x)=x\left(\xi_{0}\right)$. Then

$$
\int_{\mathcal{H}} u_{0}^{2}(x) \mu(d x)<\infty
$$

(and therefore $\sum_{m}\left(u_{m}^{0}\right)^{2}<\infty$ ). 
Proof. Recall that $A f=f^{\prime \prime}, D(A)=H^{2}(0,1) \cap H_{0}^{1}(0,1)$. By solving the two-point boundary value problem $f^{\prime \prime}=g, f(0)=f(1)=0$, after several manipulations one can show that

$$
(\Lambda h)(\xi)=\int_{0}^{1} \lambda\left(\xi, \xi^{\prime}\right) h\left(\xi^{\prime}\right) d \xi^{\prime}, \quad h \in \mathcal{H}
$$

where

$$
\lambda\left(\xi, \xi^{\prime}\right)=\frac{1}{2}\left[\left(\xi\left(1-\xi^{\prime}\right)\right)-\left(\xi-\xi^{\prime}\right) 1_{\xi^{\prime} \leq \xi}\right]
$$

The reader may more easily get convinced that this is correct a posteriori, by showing that $\frac{d^{2}}{d \xi^{2}} \int_{0}^{1} \lambda\left(\xi, \xi^{\prime}\right) f\left(\xi^{\prime}\right) d \xi^{\prime}=$ $-\frac{1}{2} f(\xi)$ and that $(\Lambda h)(0)=(\Lambda h)(1)=0$.

Consider the canonical process $\left(X_{\xi}\right)_{\xi \in[0,1]}:(\mathcal{H}, \mathcal{B}(\mathcal{H}), \mu) \rightarrow(\mathbb{R}, \mathcal{B}(\mathbb{R}))$ defined for a.e. $\xi \in[0,1]$ as $X_{\xi}(x)=x(\xi)$ and denote by $E$ the mathematical expectation on $(\mathcal{H}, \mathcal{B}(\mathcal{H}), \mu)$. The process $X$ has zero mean. One can prove that

$$
\operatorname{Cov}\left(X_{\xi}, X_{\xi^{\prime}}\right)=q\left(\xi, \xi^{\prime}\right), \quad \text { a.e. } \xi, \xi^{\prime} \in[0,1]
$$

Indeed, since $\langle\Lambda h, k\rangle_{\mathcal{H}}=\int_{\mathcal{H}}\langle x, h\rangle_{\mathcal{H}}\langle x, k\rangle_{\mathcal{H}} \mu(d x)$, we have

$$
\begin{aligned}
\int_{0}^{1} \int_{0}^{1} q\left(\xi, \xi^{\prime}\right) h\left(\xi^{\prime}\right) k(\xi) d \xi d \xi^{\prime} & =\int_{\mathcal{H}} \int_{0}^{1} x\left(\xi^{\prime}\right) h\left(\xi^{\prime}\right) d \xi^{\prime} \int_{0}^{1} x(\xi) k(\xi) d \xi \mu(d x) \\
& =\int_{0}^{1} \int_{0}^{1}\left(\int_{\mathcal{H}} x\left(\xi^{\prime}\right) x(\xi) \mu(d x)\right) h\left(\xi^{\prime}\right) k(\xi) d \xi d \xi^{\prime} \\
& =\int_{0}^{1} \int_{0}^{1} E\left[X_{\xi^{\prime}} X_{\xi}\right] h\left(\xi^{\prime}\right) k(\xi) d \xi d \xi^{\prime}
\end{aligned}
$$

and the formula for $\operatorname{Cov}\left(X_{\xi}, X_{\xi^{\prime}}\right)$ follows from the arbitrarity of $h$ and $k$.

The paths of the process $X$ are obviously of class $L^{2}(0,1)$; there is a continuous modification of $X$ if and only if $\mu$ is supported on $C([0,1])$. If we check the condition

$$
E\left[\left|X_{\xi}-X_{\xi^{\prime}}\right|^{2}\right] \leq C\left|\xi-\xi^{\prime}\right|^{\alpha}
$$

for some $\alpha, C>0$, then, by gaussianity,

$$
E\left[\left|X_{\xi}-X_{\xi^{\prime}}\right|^{p}\right] \leq C_{p}\left|\xi-\xi^{\prime}\right|^{\alpha p / 2}
$$

for every $p \geq 1$ and for a suitable constant $C_{p}>0$, hence there is a continuous modification by Kolmogorov criterion. But

$$
\begin{aligned}
E\left[\left|X_{\xi}-X_{\xi^{\prime}}\right|^{2}\right] & =E\left[X_{\xi}^{2}\right]+E\left[X_{\xi^{\prime}}^{2}\right]-2 E\left[X_{\xi} X_{\xi^{\prime}}\right] \\
& =q(\xi, \xi)+q\left(\xi^{\prime}, \xi^{\prime}\right)-2 q\left(\xi, \xi^{\prime}\right) \\
& =q(\xi, \xi)-q\left(\xi, \xi^{\prime}\right) \\
& +q\left(\xi^{\prime}, \xi^{\prime}\right)-q\left(\xi, \xi^{\prime}\right)
\end{aligned}
$$

We have

$$
\begin{aligned}
& q(\xi, \xi)-q\left(\xi, \xi^{\prime}\right) \\
& =\frac{1}{2} \xi(1-\xi)-\frac{1}{2}\left[\left(\xi\left(1-\xi^{\prime}\right)\right)-\left(\xi-\xi^{\prime}\right) 1_{\xi^{\prime} \leq \xi}\right] \\
& \leq C\left|\xi-\xi^{\prime}\right|
\end{aligned}
$$

and similarly $q\left(\xi^{\prime}, \xi^{\prime}\right)-q\left(\xi, \xi^{\prime}\right) \leq C\left|\xi-\xi^{\prime}\right|$. Hence condition (3.9) is satisfied with $\alpha=1$. We have proved that $\mu$ is supported on $C([0,1])$.

We also have

$$
\int_{\mathcal{H}} u_{0}^{2}(x) \mu(d x)=\int_{\mathcal{H}} x^{2}\left(\xi_{0}\right) \mu(d x)=E\left[X_{\xi_{0}}^{2}\right]=q\left(\xi_{0}, \xi_{0}\right)<\infty .
$$

The proof is complete. 
Remark 3.7. To convince ourselves, a more concise but a little formal proof of the claim $\int_{\mathcal{H}} u_{0}^{2}(x) \mu(d x)<$ $\infty$ is

$$
\int_{\mathcal{H}} u_{0}^{2}(x) \mu(d x)=\int_{\mathcal{H}}\left\langle x, \delta_{\xi_{0}}\right\rangle_{\mathcal{H}}^{2} \mu(d x)=\left\langle\Lambda \delta_{\xi_{0}}, \delta_{\xi_{0}}\right\rangle_{\mathcal{H}}=\frac{1}{2}\left\|(-A)^{-1 / 2} \delta_{\xi_{0}}\right\|_{\mathcal{H}}^{2}<\infty
$$

because $(-A)^{-1 / 2} \delta_{\xi_{0}} \in L^{2}(0,1)$, since by duality

$$
\begin{aligned}
\left\langle(-A)^{-1 / 2} \delta_{\xi_{0}}, f\right\rangle_{\mathcal{H}} & =\left\langle\delta_{\xi_{0}},(-A)^{-1 / 2} f\right\rangle_{\mathcal{H}}=\left((-A)^{-1 / 2} f\right)\left(\xi_{0}\right) \\
& \leq\left\|(-A)^{-1 / 2} f\right\|_{L^{\infty}} \leq C\left\|(-A)^{-1 / 2} f\right\|_{H^{1}} \leq C\|f\|_{L^{2}}
\end{aligned}
$$

where we have used Sobolev embedding $H^{1} \subset L^{\infty}$ and the fact that $(-A)^{-1 / 2}$ maps $L^{2}$ into $H^{1}$.

\section{Numerical approximation}

Define the set of finite multi-index $J^{M, N}$ as

$$
\mathcal{J}^{M, N}=\left\{\boldsymbol{\alpha}=\left(\alpha_{i}, 1 \leq i \leq M\right) \quad \mid \quad \alpha_{i} \in\{0,1,2, \ldots, N\}\right\}
$$

this is the set of $M$-tuple wich can take values in the set $\{0,1,2, \ldots, N\}$.

We approximate the solutions of the Kolmogorov equation by the following expression

$$
\hat{u}_{N}(t, x)=\sum_{\boldsymbol{n} \in \mathcal{J}^{M, N}} u_{\boldsymbol{n}}(t) H_{\boldsymbol{n}}(x), \quad x \in \mathcal{H}, \quad t \in[0, T],
$$

Notice the use of the finite $M$-tuple in oposition to the infinite multi-index $\mathcal{J}$ as in (3.5).

We truncate the infinite system (3.7) in the following sense. Consider the same value $M$ as in $J^{M, N}$ and $\boldsymbol{m}_{1}, \ldots, \boldsymbol{m}_{M} \in \mathcal{J}^{M, N}$ and define the finite system of equations

$$
\dot{u}_{\boldsymbol{m}_{i}}(t)=-u_{\boldsymbol{m}_{i}}(t) \lambda_{\boldsymbol{m}_{i}}+\sum_{j=1}^{M} u_{\boldsymbol{n}_{j}}(t) C_{\boldsymbol{n}_{j}, \boldsymbol{m}_{i}} . \quad 1 \leq i \leq M .
$$

Set the vectors

$$
\begin{aligned}
& U^{M}(t)=\left(u_{\boldsymbol{m}_{1}}(t), u_{\boldsymbol{m}_{2}}(t), \ldots, u_{\boldsymbol{m}_{M}}(t)\right)^{T} \\
& \dot{U}^{M}(t)=\left(\dot{u}_{\boldsymbol{m}_{1}}(t), \dot{u}_{\boldsymbol{m}_{2}}(t), \ldots, \dot{u}_{\boldsymbol{m}_{M}}(t)\right)^{T}
\end{aligned}
$$

and the matrix

$$
A=\left(\begin{array}{ccccc}
-\lambda_{1}+C_{1,1} & C_{2,1} & \cdots & C_{M-1,1} & C_{M, 1} \\
C_{1,2} & -\lambda_{2}+C_{2,2} & \cdots & C_{M-1,2} & C_{M, 2} \\
\vdots & \vdots & \ddots & \vdots & \vdots \\
C_{1, M-1} & C_{2, M-1} & \cdots & -\lambda_{M-1}+C_{M-1, M-1} & C_{M, M-1} \\
C_{1, M} & C_{2, M} & \cdots & C_{M-1, M} & -\lambda_{M}+C_{M, M}
\end{array}\right)
$$

where $\lambda_{i}=\lambda_{\boldsymbol{m}_{i}}$ and $C_{i, j}=C_{\boldsymbol{n}_{i}, \boldsymbol{m}_{j}}$ for $1 \leq i, j \leq M$. Notice that, given the expression (3.8), in general the matrix $A$ is not symmetric. We now can write the system (4.2) as a matrix differential equation:

$$
\dot{U}^{M}(t)=A U^{M}(t) .
$$

Then, if $A$ has $M$ real and distint eigenvalues $\eta_{i}$ and $M$ eigenvectors $\vec{V}_{i}$ then the solution to the (4.3) is given by

$$
U^{M}(t)=\sum_{i=1}^{M} c_{i} \vec{V}_{i} e^{\eta_{i} t}
$$


In the case when some of the eigenvalues and eigenvectors, or at least one of them, take values in the complex field we still can have real solutions. Indeed, Suppose that we have the case with one complex eigenvalue and eigenvector then it is know that we will have $M-2$ real eigenvalues but we can obtain two real solutions from the complex eigenvalue(see [15] for instance).

Let us write one of the complex eigenvalue and eigenvector as

$$
\begin{aligned}
\vec{V} & =\vec{a}+i \vec{b}, \\
\eta & =\gamma+i \mu,
\end{aligned}
$$

then we can write two real solutions as follows:

$$
e^{\gamma t}(\vec{a} \cos (\mu t)-\vec{b} \sin (\mu t)), \quad e^{\gamma t}(\vec{a} \sin (\mu t)+\vec{b} \cos (\mu t)) .
$$

\subsection{Initial Conditions}

In contrast to several types of differential equations, whether ordinary or partial, deterministic or stochastic, for FPK equations there is no standard way to determine the initial conditions. This is because in this type of equations we must choose a functional that acts on the initial condition, this implies that depending on the functional chosen we must adapt the method. Here we present the method for two examples of functionals.

We will consider two cases:

$$
u_{0}^{z_{0}}(g):=g\left(z_{0}\right) . \quad \text { for fixed } z_{0} \in[0,1]
$$

and

$$
u_{0}(g):=\int_{0}^{1} g(z) d z
$$

For the first functional, define the set points in the set $[a, b]$ as $\left\{z_{i}\right\}, i=0, \ldots, P$, such that $z_{0}=a$ and $z_{P}=b$. Then for each point $z_{i}$ we have that $X_{0}\left(z_{i}\right)=X\left(0, z_{i}\right)$, and for each $z_{i}$ set $u_{0}(x)$ as the evaluation functional $z_{i} \mapsto X_{t}^{x}\left(z_{i}\right)$ then from $u(t, x)=\mathbb{E}\left(u_{0}\left(X_{t}^{x}\right)\right)$ we obtain

$$
u(0, x)=\mathbb{E}\left(u_{0}^{z_{i}}\left(X_{0}^{x}\right)\right)=X^{x}\left(0, z_{i}\right)=x\left(z_{i}\right),
$$

and at other hand

$$
u(0, x)=\sum_{\boldsymbol{n} \in \mathcal{J}^{M, N}} u_{\boldsymbol{n}}(0) H_{\boldsymbol{n}}(x)
$$

then for each $z_{i}$

$$
x\left(z_{i}\right)=u(0, x)=\sum_{\boldsymbol{n} \in \mathcal{J}^{M, N}} u_{\boldsymbol{n}}(0) H_{\boldsymbol{n}}(x)
$$

Then, multiplying by $H_{\boldsymbol{m}}(x)$ and integrating in the Hilbert space $L^{2}(\mathcal{H}, \mu)$ we have

$$
u_{\boldsymbol{m}}(0)=\int_{\mathcal{H}} x\left(z_{i}\right) H_{\boldsymbol{m}}(x) \mu(d x) .
$$

Here the value of the initial condition $u_{\boldsymbol{m}}(0)$ depends on $z_{i}$, i.e. $u_{\boldsymbol{m}}(0)=u_{\boldsymbol{m}}^{z_{i}}(0)$.

Notice that in the direction of the eigenfunction $e_{k}$ the expression $x$ can be writen as $\left\langle x, e_{k}\right\rangle_{\mathcal{H}} e_{k}$ and then we can write $H_{\boldsymbol{m}}(x) x\left(z_{i}\right)$ in the direction $e_{k}$ as $P_{m_{k}}\left(\xi_{k}\right)\left\langle x, e_{k}\right\rangle_{\mathcal{H}} e_{k}\left(z_{i}\right)$ with $\xi_{k}=\left\langle x, \Lambda^{-1 / 2} e_{k}\right\rangle_{\mathcal{H}}$. Furthermore, $\xi_{k}=\left\langle x, \Lambda^{-1 / 2} e_{k}\right\rangle_{\mathcal{H}}=\left|\lambda_{k}\right|\left\langle x, e_{k}\right\rangle_{\mathcal{H}}$ then we have

$$
\begin{aligned}
u_{\boldsymbol{m}}^{z_{i}}(0) & =\int_{\mathcal{H}} x\left(z_{i}\right) H_{\boldsymbol{m}}(x) \mu(d x) \\
& =\int_{\mathbb{R}^{\mathbb{N}}} \sum_{k=1}^{\infty} e_{k}\left(z_{i}\right)\left\langle x, e_{k}\right\rangle_{\mathcal{H}} P_{m_{k}}\left(\xi_{k}\right) \mu\left(d \xi_{1}, d \xi_{2}, \cdots\right) e_{k}
\end{aligned}
$$




$$
\begin{aligned}
& =\int_{\mathbb{R}^{\mathbb{N}}} \sum_{k=1}^{\infty} e_{k}\left(z_{i}\right) \frac{\xi_{k}}{\lambda_{k}} P_{m_{k}}\left(\xi_{k}\right) \mu\left(d \xi_{1}, d \xi_{2}, \cdots\right) e_{k} \\
& =\sum_{k=1}^{\infty} \frac{e_{k}\left(z_{i}\right)}{\lambda_{k}} \int_{\mathbb{R}} P_{m_{k}}\left(\xi_{k}\right) \xi_{k} \mu\left(d \xi_{k}\right) \\
& \approx \sum_{k=1}^{M} \frac{e_{k}\left(z_{i}\right)}{\lambda_{k}} \int_{\mathbb{R}} P_{m_{k}}\left(\xi_{k}\right) \xi_{k} \mu\left(d \xi_{k}\right)
\end{aligned}
$$

Notice that the general solution to each $u_{\boldsymbol{m}}^{z_{i}}(0)$ is given by the expression

$$
\left(\begin{array}{c}
u_{1}(t) \\
u_{2}(t) \\
\vdots \\
u_{M-1}(t) \\
u_{M}(t)
\end{array}\right)=\left(\begin{array}{lllll}
\mathbf{V}_{1} & \mathbf{V}_{2} & \cdots & \mathbf{V}_{M-1} & \mathbf{V}_{M}
\end{array}\right)\left(\begin{array}{c}
c_{1} e^{\lambda_{1} t} \\
c_{2} e^{\lambda_{2} t} \\
\vdots \\
c_{M-1} e^{\lambda_{M-1} t} \\
c_{M} e^{\lambda_{M} t}
\end{array}\right)
$$

where $\mathbf{V}_{j}$ and $\lambda_{j}$ are the eigenvector and eigenvalue of the matrix $A$ and we are denoting $u_{j}(t)=$ $u_{\boldsymbol{m}_{j}}^{z_{i}}(t), 1 \leq j \leq M$. Evaluating in $t=0$ we have

and therefore

$$
\left(\begin{array}{c}
u_{1}(0) \\
u_{2}(0) \\
\vdots \\
u_{M-1}(0) \\
u_{M}(0)
\end{array}\right)=\left(\begin{array}{lllll}
\mathbf{V}_{1} & \mathbf{V}_{2} & \cdots & \mathbf{V}_{M-1} & \mathbf{V}_{M}
\end{array}\right)\left(\begin{array}{c}
c_{1} \\
c_{2} \\
\vdots \\
c_{M-1} \\
c_{M}
\end{array}\right),
$$

$$
\left(\begin{array}{c}
c_{1} \\
c_{2} \\
\vdots \\
c_{M-1} \\
c_{M}
\end{array}\right)=\left(\begin{array}{lllll}
\mathbf{V}_{1} & \mathbf{V}_{2} & \cdots & \mathbf{V}_{M-1} & \left.\mathbf{V}_{M}\right)^{-1} \\
\\
& & & \\
\vdots \\
u_{M-1}(0) \\
u_{M}(0)
\end{array}\right)
$$

with $u_{j}(t)=u_{\boldsymbol{m}_{j}}^{z_{i}}(t)$ given by the expression (4.5). Now we are able to fix the value of the initial conditions for the first case. Notice that also the contants $c_{j}$ depend on the value $z_{i}$, i.e. $c_{j}=c_{j}^{z_{i}}$.

For the second functional, from $u(t, x)=\mathbb{E}\left(u_{0}\left(X_{t}^{x}\right)\right)$ we obtain

$$
u(0, x)=\mathbb{E}\left(u_{0}\left(X_{0}^{x}\right)\right)=\int_{0}^{1} x(z) d z,
$$

and at other hand

$$
u(0, x)=\sum_{\boldsymbol{n} \in \mathcal{J}^{M, N}} u_{\boldsymbol{n}}(0) H_{\boldsymbol{n}}(x),
$$

then

$$
\int_{0}^{1} x(z) d z=\sum_{n \in \mathcal{J}^{M, N}} u_{\boldsymbol{n}}(0) H_{\boldsymbol{n}}(x) .
$$

Multiplying by $H_{\boldsymbol{m}}(x)$ and integrating in the Hilbert space $L^{2}(\mathcal{H}, \mu)$ and by using Fubini we have

$$
u_{\boldsymbol{m}}(0)=\int_{\mathcal{H}} \int_{0}^{1} x(z) d z H_{\boldsymbol{m}}(x) \mu(d x)=\int_{0}^{1}\left(\int_{\mathcal{H}} x(z) H_{\boldsymbol{m}}(x) \mu(d x)\right) d z
$$

We focus on the integral on $\mathcal{H}$. By following the steps given for the first functional (just replacing $z_{i}$ by $z$ ) we can arrive to the following expression

$$
\int_{\mathcal{H}} x(z) H_{\boldsymbol{m}}(x) \mu(d x) \approx \prod_{k=1}^{M} \frac{e_{k}(z)}{\lambda_{k}} \int_{\mathbb{R}} P_{m_{k}}\left(\xi_{k}\right) \xi_{k} \mu\left(d \xi_{k}\right),
$$


thus

$$
\begin{aligned}
u_{\boldsymbol{m}}(0) & \approx \int_{0}^{1} \prod_{k=1}^{M} \frac{e_{k}(z)}{\lambda_{k}}\left(\int_{\mathbb{R}} P_{m_{k}}\left(\xi_{k}\right) \xi_{k} \mu\left(d \xi_{k}\right)\right) d z \\
& =\prod_{k=1}^{M} \int_{\mathbb{R}} P_{m_{k}}\left(\xi_{k}\right) \xi_{k} \mu\left(d \xi_{k}\right) \int_{0}^{1} \frac{e_{k}(z)}{\lambda_{k}} d z
\end{aligned}
$$

From here and by following the procedure for the first functional we are able to fix the initial conditions.

\section{Well posedness and convergence}

Let $\mathcal{J}$ be a countable set, $\left\{\lambda_{m} ; m \in \mathcal{J}\right\}$ a sequence of positive real numbers diverging to infinity and $\left\{C_{n m} ; n, m \in \mathcal{J}\right\}$ a sequence of real numbers. Consider the infinite system of equations

$$
\begin{aligned}
& u_{m}^{\prime}(t)=-\lambda_{m} u_{m}(t)+\sum_{n \in \mathcal{J}} C_{n m} u_{n}(t), \quad t \geq 0 \\
& u_{m}(0)=u_{m}^{0}, \quad m \in \mathcal{J}
\end{aligned}
$$

with given initial condition $\left\{u_{m}^{0} ; m \in \mathcal{J}\right\}$. We always assume

$$
\sum_{m \in \mathcal{J}}\left(u_{m}^{0}\right)^{2}<\infty
$$

Definition 5.1. A solution is a sequence $\left\{u_{m}(\cdot) ; m \in \mathcal{J}\right\}$ of continuous functions on $[0, T]$ such that: i)

$$
\sup _{t \in[0, T]} \sum_{m \in \mathcal{J}} u_{m}^{2}(t)+\int_{0}^{T} \sum_{m \in \mathcal{J}} \lambda_{m} u_{m}^{2}(s) d s<\infty
$$

ii) the series $\sum_{n \in \mathcal{J}} C_{n m} u_{n}(t)$ converges, for a.e. $t$, to an integrable functions on $[0, T]$ and iii)

$$
u_{m}(t)=u_{m}^{0}-\int_{0}^{t} \lambda_{m} u_{m}(s) d s+\int_{0}^{t} \sum_{n \in \mathcal{J}} C_{n m} u_{n}(s) d s
$$

for all $m \in \mathcal{J}$ and $t \in[0, T]$.

Consider also, for any finite subset $\widetilde{\mathcal{J}} \subset \mathcal{J}$, the finite system

$$
\begin{aligned}
& \widetilde{u}_{m}^{\prime}(t)=-\lambda_{m} \widetilde{u}_{m}(t)+\sum_{n \in \widetilde{\mathcal{J}}} C_{n m} \widetilde{u}_{n}(t), \quad t \geq 0 \\
& \widetilde{u}_{m}(0)=u_{m}^{0}, \quad m \in \mathcal{\mathcal { J }}
\end{aligned}
$$

The definition of solution for this finite system is obvious and existence and uniqueness is well known.

Theorem 5.2. Assume that the family $\left\{C_{n m} ; n, m \in \mathcal{J}\right\}$ satisfies, for some constant $C>0$,

$$
\sum_{n, m \in \mathcal{J}} C_{n m} \alpha_{n} \beta_{m} \leq C\left(\sum_{n \in \mathcal{J}} \lambda_{n} \alpha_{n}^{2}\right)^{1 / 2}\left(\sum_{m \in \mathcal{J}} \beta_{m}^{2}\right)^{1 / 2} \quad \text { for all sequences }\left\{\alpha_{n}, \beta_{n} ; n \in \mathcal{J}\right\} \text {. }
$$

Then there exists a unique solution. Moreover,

$$
\sup _{t \in[0, T]} \sum_{m \in \widetilde{\mathcal{J}}}\left(u_{m}(t)-\widetilde{u}_{m}(t)\right)^{2}+\int_{0}^{T} \sum_{m \in \widetilde{\mathcal{J}}} \lambda_{m}\left(u_{m}(s)-\widetilde{u}_{m}(s)\right)^{2} d s \leq C_{1} \int_{0}^{T} \sum_{m \in \widetilde{\mathcal{J}}^{c}} \lambda_{m} u_{m}^{2}(s) d s
$$

for some $C_{1}>0$ independent of $\widetilde{\mathcal{J}}$; where the term $\int_{0}^{T} \sum_{m \in \widetilde{J}^{c}} \lambda_{m} u_{m}^{2}(s) d s$ converges to zero as $\widetilde{\mathcal{J}}$ converges to $\mathcal{J}$. 
Remark 5.3. Under assumption (5.1), given $m_{0} \in \mathcal{J}$ and $s \in[0, T]$, choose $\alpha_{n}=u_{n}(s)$ and $\beta_{n}$ equal to zero except for $\beta_{m_{0}}=1$; then

$$
\left|\sum_{n \in \mathcal{J}} C_{n m_{0}} u_{n}(s)\right|=\left|\sum_{n, m \in \mathcal{J}} C_{n m} u_{n}(s) \beta_{m}\right| \leq C\left(\sum_{n \in \mathcal{J}} \lambda_{n} u_{n}^{2}(s)\right)^{1 / 2} \leq C\left(1+\sum_{n \in \mathcal{J}} \lambda_{n} u_{n}^{2}(s)\right)
$$

hence, in Definition 5.1. condition (i) implies (ii).

Proof. Step 1 (existence and uniqueness). Let $H, V$ be the real separable Hilbert spaces of sequences $\alpha=\left\{\alpha_{n} ; n \in \mathcal{J}\right\}$ such that, respectively $\|\alpha\|_{H}^{2}:=\sum_{n \in \mathcal{J}} \alpha_{n}^{2}<\infty,\|\alpha\|_{V}^{2}:=\sum_{n \in \mathcal{J}} \lambda_{n} \alpha_{n}^{2}<\infty$, with norms $\|\alpha\|_{H}^{2}$ and $\|\alpha\|_{V}^{2}$ respectively; let $\langle\cdot, \cdot\rangle_{H}$ denote the inner product in $H$. Since we have assumed at the beginning that $\left\{\lambda_{m} ; m \in \mathcal{J}\right\}$ diverges to infinity, we have $V \subset H$ and there exists a constant $C_{H, V}$ such that $\|\alpha\|_{H}^{2} \leq C_{H, V}\|\alpha\|_{V}^{2}$ for all $\alpha \in V$. Let $V^{\prime}$ be the dual space of $V$, with norm $\|\cdot\|_{V^{\prime}}^{2}$. We identify $H$ with its dual $H^{\prime}$ so that $V \subset H \subset V^{\prime}$ and denote by $\langle\cdot, \cdot\rangle$ the dual pairing between $V$ and $V^{\prime}$, which extends $\langle\cdot, \cdot\rangle_{H}$.

Let $a(\cdot, \cdot): V \times V \rightarrow \mathbb{R}$ be the bilinear map defined as

$$
a(\alpha, \beta)=\sum_{n \in \mathcal{J}} \lambda_{n} \alpha_{n} \beta_{n}-\sum_{n, m \in \mathcal{J}} C_{n m} \alpha_{n} \beta_{m} .
$$

It holds

$$
\begin{aligned}
|a(\alpha, \beta)| & \leq \sum_{n \in \mathcal{J}} \lambda_{n} \alpha_{n}^{2}+\sum_{n \in \mathcal{J}} \lambda_{n} \beta_{n}^{2}+C\left(\sum_{n \in \mathcal{J}} \lambda_{n} \alpha_{n}^{2}\right)^{1 / 2}\left(\sum_{n \in \mathcal{J}} \beta_{n}^{2}\right)^{1 / 2} \\
& =(1+C)\|\alpha\|_{V}^{2}+\|\beta\|_{V}^{2}+C\|\beta\|_{H}^{2}
\end{aligned}
$$

hence $a(\cdot, \cdot)$ is well defined and continuous on $V \times V$. Moreover, since

$$
C\left(\sum_{n \in \mathcal{J}} \lambda_{n} \alpha_{n}^{2}\right)^{1 / 2}\left(\sum_{n \in \mathcal{J}} \beta_{n}^{2}\right)^{1 / 2} \leq \frac{1}{2} \sum_{n \in \mathcal{J}} \lambda_{n} \alpha_{n}^{2}+2 C^{2} \sum_{n \in \mathcal{J}} \beta_{n}^{2}
$$

we get

$$
a(\alpha, \alpha)=\sum_{n \in \mathcal{J}} \lambda_{n} \alpha_{n}^{2}-\sum_{n, m \in \mathcal{J}} C_{n m} \alpha_{n} \alpha_{m} \geq \frac{1}{2} \sum_{n \in \mathcal{J}} \lambda_{n} \alpha_{n}^{2}-2 C^{2}\|\alpha\|_{H}^{2}
$$

hence $a(\cdot, \cdot)$ is coercive on $V \times V$. Consider the equation

$$
\langle u(t), \phi\rangle_{H}+\int_{0}^{t} a(u(s), \phi) d s=\left\langle u^{0}, \phi\right\rangle_{H}+\int_{0}^{t}\langle f(s), \phi\rangle d s
$$

with $\phi \in V, u^{0} \in H, f \in L^{2}(0, T ; H)$ (one can treat also $f \in L^{2}\left(0, T ; V^{\prime}\right)$ but this is not important here). By solution we mean a function $u \in L^{\infty}(0, T ; H) \cap L^{2}(0, T ; V)$ which satisfies this equation for all $\phi \in V$ and all $t \in[0, T]$. By a well known theorem (see [23]), there exists a unique solution of this equation, with

$$
\sup _{t \in[0, T]}\|u(t)\|_{H}^{2}+\int_{0}^{T}\|u(s)\|_{V}^{2} d s<\infty .
$$

This proves existence and uniqueness of a solution of the infinite system above, in the sense of Definition 5.1 .

Step 2 (convergence) Let us prove the estimate between the finite and infinite system. We have

$$
u_{m}(t)=u_{m}^{0}-\int_{0}^{t} \lambda_{m} u_{m}(s) d s+\int_{0}^{t} \sum_{n \in \widetilde{\mathcal{J}}} C_{n m} u_{n}(s) d s+\int_{0}^{t} R_{m}^{\widetilde{\mathcal{J}}}(s) d s
$$


where $R_{m}^{\widetilde{\mathcal{J}}}(s)=\sum_{n \in \widetilde{\mathcal{J}}^{c}} C_{n m} u_{n}(s)$; we know that $R_{m}^{\widetilde{\mathcal{J}}}$ is an integrable function, by definition of solution. Then, for the new variable $v_{m}(t):=u_{m}(t)-\widetilde{u}_{m}(t)$ we have

$$
v_{m}(t)=-\int_{0}^{t} \lambda_{m} v_{m}(s) d s+\int_{0}^{t} \sum_{n \in \widetilde{\mathcal{J}}} C_{n m} v_{n}(s) d s+\int_{0}^{t} R_{m}^{\widetilde{\mathcal{J}}}(s) d s .
$$

It follows that the family $\left\{v_{m} ; m \in \tilde{\mathcal{J}}\right\}$ satisfies the finite system

$$
\begin{aligned}
& v_{m}^{\prime}(t)=-\lambda_{m} v_{m}(t)+\sum_{n \in \widetilde{\mathcal{J}}} C_{n m} v_{n}(t)+R_{m}^{\widetilde{\mathcal{J}}}(t), \quad t \geq 0 \\
& v_{m}(0)=0, \quad m \in \widetilde{\mathcal{J}} .
\end{aligned}
$$

We have

$$
\begin{aligned}
\sum_{m \in \widetilde{\mathcal{J}}} v_{m} R_{m}^{\widetilde{\mathcal{J}}} & =\sum_{m \in \widetilde{\mathcal{J}}} \sum_{n \in \widetilde{\mathcal{J}}^{c}} C_{n m} u_{n} v_{m} \leq C\left(\sum_{n \in \widetilde{\mathcal{J}}^{c}} \lambda_{n} u_{n}^{2}\right)^{1 / 2}\left(\sum_{n \in \widetilde{\mathcal{J}}} v_{n}^{2}\right)^{1 / 2} \\
& \leq C^{2} \sum_{n \in \widetilde{\mathcal{J}}} v_{n}^{2}+\sum_{n \in \widetilde{\mathcal{J}}^{c}} \lambda_{n} u_{n}^{2}
\end{aligned}
$$

and thus

$$
\begin{aligned}
\frac{1}{2} \frac{d}{d t} \sum_{m \in \widetilde{\mathcal{J}}} v_{m}^{2}+\sum_{m \in \widetilde{\mathcal{J}}} \lambda_{m} v_{m}^{2} & =\sum_{n, m \in \widetilde{\mathcal{J}}} C_{n m} v_{n} v_{m}+\sum_{m \in \widetilde{\mathcal{J}}} v_{m} R_{m}^{\widetilde{\mathcal{J}}} \\
& \leq C\left(\sum_{n \in \mathcal{J}} \lambda_{n} v_{n}^{2}\right)^{1 / 2}\left(\sum_{n \in \mathcal{J}} v_{n}^{2}\right)^{1 / 2}+C^{2} \sum_{n \in \widetilde{\mathcal{J}}_{n}} v_{n}^{2} \sum_{n \in \widetilde{\mathcal{J}}^{c}} \lambda_{n} u_{n}^{2} \\
& \leq \frac{1}{2} \sum_{m \in \widetilde{\mathcal{J}}} \lambda_{m} v_{m}^{2}+3 C^{2} \sum_{m \in \widetilde{\mathcal{J}}} v_{m}^{2}+\sum_{n \in \widetilde{\mathcal{J}}^{c}} \lambda_{n} u_{n}^{2}
\end{aligned}
$$

hence (renaming the constant $C$ )

$$
\frac{1}{2} \frac{d}{d t} \sum_{m \in \widetilde{\mathcal{J}}} v_{m}^{2}+\frac{1}{2} \sum_{m \in \widetilde{\mathcal{J}}} \lambda_{m} v_{m}^{2} \leq 3 C^{2} \sum_{m \in \widetilde{\mathcal{J}}} v_{m}^{2}+\sum_{n \in \widetilde{\mathcal{J}}^{c}} \lambda_{n} u_{n}^{2}
$$

which, by Gronwall lemma, easily implies that there exists a constant $C_{1}>0$, independent of the finite subset $\mathcal{J}$, such that

$$
\sup _{t \in[0, T]} \sum_{m \in \widetilde{\mathcal{J}}} v_{m}^{2}(t)+\int_{0}^{T} \sum_{m \in \widetilde{\mathcal{J}}} \lambda_{m} v_{m}^{2}(s) d s \leq C_{1} \int_{0}^{T} \sum_{n \in \widetilde{\mathcal{J}}^{c}} \lambda_{n} u_{n}^{2}(s) d s .
$$

The proof is complete.

Proposition 5.4. Let $B: \mathcal{H} \rightarrow \mathcal{H}$ be bounded measurable and let $C_{n m}$ be given by

$$
C_{n m}=\int_{\mathcal{H}}\left\langle B(x), D_{x} H_{n}(x)\right\rangle_{\mathcal{H}} H_{m}(x) \mu(d x) .
$$

If

$$
\int_{\mathcal{H}}\left|D_{x} \varphi(x)\right|_{\mathcal{H}}^{2} \mu(d x) \leq 2 \sum_{n \in \mathcal{J}} \lambda_{n} \varphi_{n}^{2}
$$

for every function $\varphi(x)$ of the form $\varphi(x)=\sum_{n \in \mathcal{J}} \varphi_{n} H_{n}(x)$, then condition (5.1) holds true. 
Proof. Given two sequences $\left\{\alpha_{n}, \beta_{n} ; n \in \mathcal{J}\right\}$, setting

$$
\varphi(x)=\sum_{n \in \mathcal{J}} \alpha_{n} H_{n}(x), \quad \psi(x)=\sum_{m \in \mathcal{J}} \beta_{m} H_{m}(x)
$$

one simply has

$$
\begin{aligned}
\sum_{n, m \in \mathcal{J}} C_{n m} \alpha_{n} \beta_{m} & =\int_{\mathcal{H}}\left\langle B(x), D_{x} \varphi(x)\right\rangle_{\mathcal{H}} \psi(x) \mu(d x) \\
& \leq\|B\|_{\infty} \int_{\mathcal{H}}\left|D_{x} \varphi(x)\right|_{\mathcal{H}}|\psi(x)| \mu(d x) \\
& \leq\|B\|_{\infty}\left(\int_{\mathcal{H}}\left|D_{x} \varphi(x)\right|_{\mathcal{H}}^{2} \mu(d x)\right)^{1 / 2}\left(\int_{\mathcal{H}}|\psi(x)|^{2} \mu(d x)\right)^{1 / 2} \\
& \leq\|B\|_{\infty}\left(2 \sum_{n \in \mathcal{J}} \lambda_{n} \alpha_{n}^{2}\right)^{1 / 2}\left(\sum_{m \in \mathcal{J}} \beta_{m}^{2}\right)^{1 / 2}
\end{aligned}
$$

Now, we will prove that $(5.2)$ is satisfied in our case. Assume the conditions in Lemma 3.4 holds. Then, for any $\Phi, \Psi \in \mathcal{S}(\mathbb{H})^{1}$ the following Green's formula holds (for a proof see Lemma 4.4 in [6] for instance)

$$
-\frac{1}{2} \int_{\mathcal{H}}\left\langle Q D_{x} \Phi, D_{x} \Psi\right\rangle_{\mathcal{H}} \mu(d x)=\int_{\mathcal{H}}\left(\mathcal{A}_{0} \Phi\right) \Psi \mu(d x)=\int_{\mathcal{H}} \Phi\left(\mathcal{A}_{0} \Psi\right) \mu(d x) .
$$

By taking $\Psi=\Phi=\varphi$ and $Q=I d$ we have

$$
\int_{\mathcal{H}}\left|D_{x} \varphi\right|_{\mathcal{H}}^{2} \mu(d x)=\int_{\mathcal{H}}\left\langle D_{x} \varphi, D_{x} \varphi\right\rangle_{\mathcal{H}} \mu(d x)=-2 \int_{\mathcal{H}}\left(\mathcal{A}_{0} \varphi\right) \varphi \mu(d x)
$$

If $\varphi(x)=\sum_{n \in \mathcal{J}} \varphi_{n} H_{n}(x)$, then

$$
\begin{aligned}
-\int_{\mathcal{H}}\left(\mathcal{A}_{0} \varphi\right) \varphi \mu(d x) & =\int_{\mathcal{H}}\left(-\mathcal{A}_{0} \sum_{n \in \mathcal{J}} \varphi_{n} H_{n}(x)\right) \sum_{m \in \mathcal{J}} \varphi_{m} H_{m}(x) \mu(d x) \\
& =\sum_{m \in \mathcal{J}} \int_{\mathcal{H}}\left(\sum_{n \in \mathcal{J}} \varphi_{n}\left[-\mathcal{A}_{0} H_{n}(x)\right]\right) \varphi_{m} H_{m}(x) \mu(d x) \\
& =\sum_{m \in \mathcal{J}} \int_{\mathcal{H}} \sum_{n \in \mathcal{J}} \varphi_{n} \lambda_{n} H_{n}(x) \varphi_{m} H_{m}(x) \mu(d x) \\
& =\sum_{m \in \mathcal{J}} \sum_{n \in \mathcal{J}} \varphi_{n} \varphi_{m} \lambda_{n} \int_{\mathcal{H}} H_{n}(x) H_{m}(x) \mu(d x) \\
& =\sum_{n \in \mathcal{J}} \lambda_{n} \varphi_{n}^{2} .
\end{aligned}
$$

Where in the last step we will use that $H_{n}(x)$ is an orthonormal basis for $\mathcal{H}$. Then, we have

$$
\int_{\mathcal{H}}\left|D_{x} \varphi\right|_{\mathcal{H}}^{2} \mu(d x)=-2 \int_{\mathcal{H}}\left(\mathcal{A}_{0} \varphi\right) \varphi \mu(d x)=2 \sum_{n \in \mathcal{J}} \lambda_{n} \varphi_{n}^{2} .
$$

\footnotetext{
${ }^{1}$ Recall that $\mathcal{S}(\mathbb{H})$ is the set of all cylinder functionals on $\mathcal{H}$
} 


\section{Numerical Results}

\subsection{Algorithm description}

In this subsection we describe the algorithm we follow to get the simulations for the Kolmogorov equations associated with three stochastic partial differential equations whose results we show in next subsections.

1. Choose the algorithm's parameters:

a) The space $\mathcal{H}$ where the SPDE will be defined.

b) The operator $A$ and its eigenfunctions $\lambda_{k}$ and eigenvalues $e_{k}(\cdot)$.

c) The functional $u_{0}: \mathcal{H} \rightarrow \mathbb{R}$.

d) $N, M$ and then fix the set $J^{N, M}$.

e) The time step $\Delta t$ and $\Delta x$ in the physical space.

2. Compute the quantities $\bar{C}_{\boldsymbol{n}, \boldsymbol{m}}$, for each $\boldsymbol{n}, \boldsymbol{m} \in J^{N, M}$, to approximate (3.8).

3. Set the finite system of coupled ordinary differential equation (4.2)

4. Rewriting the system (4.2) as a matrix differential equations and by solving it numerically we obtain, up to a set of constants, the time-functions $u_{\boldsymbol{n}}(t)$, for each $\boldsymbol{n} \in J^{N, M}$.

5. By using the functional $u_{0}$ the constants in the last step are fixed.

6. We then define the space-time approximation for the Kolmogorov equation as

$$
u_{N}(t, x)=\sum_{j=1}^{N} u_{j}(t) H_{j}(x) \approx \sum_{j \geq 1} u_{j}(t) H_{j}(x)=u(t, x)
$$

Remark 6.1. - Given the operator A, we choose its eigenvalues as the basis for the Hilbert space $\mathcal{H}$ and we have to find its eigenvalues $\lambda_{k}$.

- The choice of the functional $u_{0}$ will change the way we determine the initial condition of the Kolmogorov equation, then it will necessary to adapt the method for each $u_{0}$.

- the quantities $\bar{C}_{n, m}$ are those that require more computing resources because we have to compute and approximate several integrals for each $\boldsymbol{n}, \boldsymbol{m} \in J^{N, M}$. In our examples these quantities are given by the expressions (6.5), (6.14) and (6.21).

\subsection{Stochastic Heat equation in an interval}

As a first application consider the stochastic diffusion in dimension 1 .

Let $\mathcal{H}=L^{2}([0,1]), Q=I d$, and $A$ be given by $A x=\nu \triangle_{\xi} x, x \in D(A)$ with $D(A)=H^{2}(0,1) \cap$ $H_{0}^{1}(0,1)$ (where $H^{2}(0,1)$ is the Sobolev spaces and $H_{0}^{1}(0,1)$ is the subspace of $H^{1}(0,1)$ of all functions vanishing at 0,1$)$.

Consider the heat equation in $[0,1]$

$$
\begin{aligned}
\frac{\partial X(t, \xi)}{\partial t} & =\nu \frac{\partial^{2} X(t, \xi)}{\partial \xi^{2}}+f(\xi)+\frac{\partial^{2} W}{\partial t \partial \xi}, \quad \xi \in[0,1] \\
\left.X(t, \xi)\right|_{t=0} & =X_{0}(\xi), \quad X_{0} \in \mathcal{H}, \\
X(t, \xi) & =0, \quad t \geq 0, \xi=0,1,
\end{aligned}
$$


where $t \in[0, T], f(\xi)=\xi^{3}, X_{0}(\xi)=\sin (\pi x) . W$ is a cylindrical Wiener process on $\mathcal{H}$, associated to a stochastic basis $\left(\Omega, \mathcal{F}, \mathbb{P},\left(\mathcal{F}_{t}\right)_{t \geq 0}\right)$. $\nu$ denotes the thermal diffusivity.

The complete orthonormal system of eigenfunctions $e_{k}$ is defined as

$$
e_{k}(\xi)=\sqrt{2} \sin (k \pi \xi), \quad \xi \in[0,1], \quad k \in \mathbb{N} .
$$

$\mathrm{A}$ is self-adjoint negative operator and $A e_{k}=-\nu k^{2} \pi^{2} e_{k}, k \in \mathbb{N}$.

We rewrite the equation (6.1) as an abstract differential equation on $\mathcal{H}$. Set $B=f$, then

$$
\begin{aligned}
d X & =[A X+B(X)] d t+d W_{t}, \\
X(0) & =x, \quad x \in \mathcal{H}
\end{aligned}
$$

Define $u(t, x)=\mathbb{E}\left[u_{0}\left(X_{t}^{x}\right)\right]$ and then $u(t, x)$ satisfies the Kolmogorov equation

$$
\frac{\partial u}{\partial t}=\frac{1}{2} \operatorname{Tr}\left(Q D^{2} u\right)+\langle A x, D u\rangle_{\mathcal{H}}+\langle B(x), D u\rangle_{\mathcal{H}}, \quad x \in D(A) .
$$

We will consider two cases :

and

$$
u_{0}^{\xi_{0}}(g):=g\left(\xi_{0}\right) . \quad \text { for fixed } \xi_{0} \in(0,1)
$$

$$
u_{0}(g):=\int_{0}^{1} g(\xi) d \xi
$$

As before we write the solution as

$$
u(t, x)=\sum_{\boldsymbol{n} \in \mathcal{J}} u_{\boldsymbol{n}}(t) H_{\boldsymbol{n}}(x), \quad x \in \mathcal{H}, \quad t \in[0, T],
$$

where $u_{n}:[0, T] \mapsto \mathbb{R}$ and $H_{n}(x)$ are the Hermite functionals. Following the last procedure we set the infinite system of coupled ordinary differential equations.

$$
\dot{u}_{\boldsymbol{m}}(t)=-u_{\boldsymbol{m}}(t) \lambda_{\boldsymbol{m}}+\sum_{\boldsymbol{n} \in \mathcal{J}} u_{\boldsymbol{n}}(t) C_{\boldsymbol{n}, \boldsymbol{m}}, \quad \boldsymbol{n}, \boldsymbol{m} \in \mathcal{J}
$$

where $C_{n, m}$ is given by

$$
C_{\boldsymbol{n}, \boldsymbol{m}}:=\int_{\mathcal{H}}\left\langle B(x), D_{x} H_{\boldsymbol{n}}(x)\right\rangle_{\mathcal{H}} H_{\boldsymbol{m}}(x) \mu(d x) .
$$

The numerical method for this case is applied now. We have that $\Lambda=\frac{1}{2}(-A)^{-1}$ have eigenvalues $1 /\left(2 \nu \pi^{2}|k|^{2}\right)$, then the operator $\Lambda^{-1}$ is well-defined and have eigenvalues $2 \nu \pi^{2}|k|^{2}$, and $\Lambda^{-\frac{1}{2}}$ can also be befined having eigenvalues $\sqrt{2 \nu} \pi|k|$, then

$$
\left\langle B(x), \Lambda^{-\frac{1}{2}} e_{k}\right\rangle_{L^{2}([0,1])}=\sqrt{2 \nu} \pi|k|\left\langle f, e_{k}\right\rangle_{L^{2}([0,1])} .
$$

Notice that $H_{\boldsymbol{n}}=\prod_{\alpha} P_{n_{\alpha}}\left(\xi_{\alpha}\right), H_{\boldsymbol{m}}=\prod_{\alpha} P_{m_{\alpha}}\left(\xi_{\alpha}\right)$ and $P_{m_{k}}^{\prime}\left(\xi_{k}\right)=m_{k}^{1 / 2} P_{m_{k}-1}\left(\xi_{k}\right)$. Then, we rewrite $C_{n, m}$ as follows.

$$
\begin{aligned}
C_{\boldsymbol{n}, \boldsymbol{m}} & =\sum_{k=1}^{\infty} \int_{\mathcal{H}}\left\langle B(x), \Lambda^{-\frac{1}{2}} e_{k}\right\rangle_{\mathcal{H}} \prod_{\substack{i=1 \\
i \neq k}}^{\infty} P_{n_{i}}\left(\left\langle x, \Lambda^{-\frac{1}{2}} e_{i}\right\rangle_{\mathcal{H}}\right) P_{n_{k}}^{\prime}\left(\left\langle x, \Lambda^{-\frac{1}{2}} e_{k}\right\rangle_{\mathcal{H}}\right) H_{\boldsymbol{m}}(x) \mu(d x) \\
& =\sum_{k=1}^{\infty} \int_{\mathcal{H}} \lambda_{k}\left\langle f, e_{k}\right\rangle_{\mathcal{H}} P_{m_{k}}\left(\left\langle x, \Lambda^{-\frac{1}{2}} e_{k}\right\rangle_{\mathcal{H}}\right) P_{n_{k}}^{\prime}\left(\left\langle x, \Lambda^{-\frac{1}{2}} e_{k}\right\rangle_{\mathcal{H}}\right)
\end{aligned}
$$




$$
\times \prod_{\substack{i=1 \\ i \neq k}}^{\infty} P_{n_{i}}\left(\left\langle x, \Lambda^{-\frac{1}{2}} e_{i}\right\rangle_{\mathcal{H}}\right) P_{m_{i}}\left(\left\langle x, \Lambda^{-\frac{1}{2}} e_{i}\right\rangle_{\mathcal{H}}\right) \mu(d x) .
$$

Writing the measure $\mu(d x)$ in the direction $e_{k}$ as $\mu(d x) e_{k}=\frac{1}{\lambda_{k}} \mu\left(d\left(\left\langle x, \Lambda^{-\frac{1}{2}} e_{k}\right\rangle_{\mathcal{H}}\right)\right)=\frac{1}{\lambda_{k}} \mu\left(d \xi_{k}\right)$ with $\xi_{k}=\left\langle x, \Lambda^{-\frac{1}{2}} e_{k}\right\rangle_{\mathcal{H}}$, then we approximate $C_{\boldsymbol{n}, \boldsymbol{m}}$ as

$$
\begin{aligned}
C_{\boldsymbol{n}, \boldsymbol{m}}= & \sum_{k=1}^{\infty} \lambda_{k} \int_{0}^{1} f(\xi) e_{k}(\xi) d \xi \int_{\mathbb{R}} n_{k}^{1 / 2} P_{m_{k}}\left(\xi_{k}\right) P_{n_{k}-1}\left(\xi_{k}\right) \frac{1}{\lambda_{k}} \mu\left(d \xi_{k}\right) \\
& \times \int_{\mathbb{R}^{\mathbb{N}}} \prod_{\substack{i=1 \\
i \neq k}}^{\infty} P_{n_{i}}\left(\xi_{i}\right) P_{m_{i}}\left(\xi_{i}\right) \frac{1}{\lambda_{i}} \mu\left(d \xi_{i}\right) \\
\approx & \sum_{k=1}^{M} \int_{0}^{1} f(\xi) e_{k}(\xi) d \xi \int_{\mathbb{R}} n_{k}^{1 / 2} P_{m_{k}}\left(\xi_{k}\right) P_{n_{k}-1}\left(\xi_{k}\right) \mu\left(d \xi_{k}\right) \\
& \times \int_{\mathbb{R}^{M-1}} \prod_{\substack{i=1 \\
i \neq k}}^{M} P_{n_{i}}\left(\xi_{i}\right) P_{m_{i}}\left(\xi_{i}\right) \frac{1}{\lambda_{i}} \mu\left(d \xi_{i}\right) \\
= & \sum_{k=1}^{M} n_{k}^{1 / 2} \int_{0}^{1} f(\xi) e_{k}(\xi) d \xi \int_{\mathbb{R}} P_{m_{k}}\left(\xi_{k}\right) P_{n_{k}-1}\left(\xi_{k}\right) \mu\left(d \xi_{k}\right) \\
& \times \prod_{\substack{i=1 \\
i \neq k}}^{M} \frac{1}{\lambda_{i}} \int_{\mathbb{R}} P_{n_{i}}\left(\xi_{i}\right) P_{m_{i}}\left(\xi_{i}\right) \mu\left(d \xi_{i}\right)
\end{aligned}
$$

For $N_{1} \in \mathbb{N}$ define the set $S_{N_{1}}=\left\{\boldsymbol{n}_{1}, \boldsymbol{n}_{2}, \ldots, \boldsymbol{n}_{N_{1}}: \boldsymbol{n}_{i} \in J^{M, N}, i=1, \ldots, N_{1}\right\}$. Moreover, for $\boldsymbol{n}, \boldsymbol{m} \in S_{M}$ define

$$
\begin{aligned}
\bar{C}_{\boldsymbol{n}, \boldsymbol{m}}:=\sum_{k=1}^{M} & \sqrt{2 \nu} \pi|k| n_{k}^{1 / 2} \int_{0}^{1} f(\xi) e_{k}(\xi) d \xi \int_{\mathbb{R}} P_{m_{k}}\left(\xi_{k}\right) P_{n_{k}-1}\left(\xi_{k}\right) \mu\left(d \xi_{k}\right) \\
& \times \prod_{\substack{i=1 \\
i \neq k}}^{M} \int_{\mathbb{R}} P_{n_{i}}\left(\xi_{i}\right) P_{m_{i}}\left(\xi_{i}\right) \mu\left(d \xi_{i}\right),
\end{aligned}
$$

and the finite system of ordinary differential equations:

$$
\dot{u}_{\boldsymbol{m}}(t)=-u_{\boldsymbol{m}}(t) \lambda_{\boldsymbol{m}}+\sum_{\boldsymbol{n} \in S_{M}} u_{\boldsymbol{n}}(t) \bar{C}_{\boldsymbol{n}, \boldsymbol{m}}, \quad \text { for each } \boldsymbol{m} \in S_{M} \text { and } \boldsymbol{n} \in S_{M} .
$$

Then (6.6) approximates to the infinite system of ordinary differential equations (6.3) when $N, M \rightarrow \infty$. We use the system (6.6) to approximate the solution of the FPK equation associated with the Diffusion equation.

We need to evaluate the integrals and do the finite sum on $k$, to do this we use a Gauss-Hermite quadrature to approximate the value of the integrals

$$
\int_{0}^{1} f(\xi) e_{k}(\xi) d \xi, \quad \int_{\mathbb{R}} P_{m_{k}}\left(\xi_{k}\right) P_{n_{k}-1}\left(\xi_{k}\right) \mu\left(d \xi_{k}\right), \quad \int_{\mathbb{R}} P_{n_{i}}\left(\xi_{i}\right) P_{m_{i}}\left(\xi_{i}\right) \mu\left(d \xi_{i}\right) .
$$

When the constans $C_{n, m}$ are fixed we solve the Matrix Differential equation (4.3):

$$
\dot{U}^{M}(t)=A U^{M}(t) .
$$


with

$$
A=\left(\begin{array}{ccccc}
-\lambda_{1}+C_{1,1} & C_{2,1} & \cdots & C_{M-1,1} & C_{M, 1} \\
C_{1,2} & -\lambda_{2}+C_{2,2} & \cdots & C_{M-1,2} & C_{M, 2} \\
\vdots & \vdots & \ddots & \vdots & \vdots \\
C_{1, M-1} & C_{2, M-1} & \cdots & -\lambda_{M-1}+C_{M-1, M-1} & C_{M, M-1} \\
C_{1, M} & C_{2, M} & \cdots & C_{M-1, M} & -\lambda_{M}+C_{M, M}
\end{array}\right)
$$

$\lambda_{i}=\lambda_{\boldsymbol{m}_{i}}$ and $C_{i, j}=C_{\boldsymbol{n}_{i}, \boldsymbol{m}_{j}}$ for $1 \leq i, j \leq M$, and

$$
\begin{aligned}
& U^{M}(t)=\left(u_{\boldsymbol{m}_{1}}(t), u_{\boldsymbol{m}_{2}}(t), \ldots, u_{\boldsymbol{m}_{M}}(t)\right)^{T} \\
& \dot{U}^{M}(t)=\left(\dot{u}_{\boldsymbol{m}_{1}}(t), \dot{u}_{\boldsymbol{m}_{\mathbf{2}}}(t), \ldots, \dot{u}_{\boldsymbol{m}_{M}}(t)\right)^{T}
\end{aligned}
$$

From this we get the general solution of (6.7) is given by

$$
\left(\begin{array}{c}
u_{1}(t) \\
u_{2}(t) \\
\vdots \\
u_{M-1}(t) \\
u_{M}(t)
\end{array}\right)=\left(\begin{array}{lllll}
\mathbf{V}_{1} & \mathbf{V}_{2} & \cdots & \mathbf{V}_{M-1} & \mathbf{V}_{M}
\end{array}\right)\left(\begin{array}{c}
c_{1} e^{\lambda_{1} t} \\
c_{2} e^{\lambda_{2} t} \\
\vdots \\
c_{M-1} e^{\lambda_{M-1} t} \\
c_{M} e^{\lambda_{M} t}
\end{array}\right)
$$

where $\mathbf{V}_{i}$ and $\lambda_{i}$ are the eigenvector and eigenvalue of the matrix $A$. It remains to fix the set of constants $\left\{c_{i}, 1 \leq i \leq M\right\}$ which are determined by using the initial conditions given in subsection 4.1.

\section{Initial Conditions}

We define the set points in the set $[0,1]$ as $\left\{\xi_{i}\right\}, i=0, \ldots, P$, such that $\xi_{0}=0$ and $\xi_{P}=1$. Then by using (4.5) we fix the values of the constants $c_{i}$

$$
\left(\begin{array}{c}
c_{1} \\
c_{2} \\
\vdots \\
c_{M}
\end{array}\right)=\left[\left(\begin{array}{cccc}
H_{1}\left(x_{0}\right) & H_{2}\left(x_{0}\right) & \cdots & H_{m}\left(x_{0}\right) \\
H_{1}\left(x_{1}\right) & H_{2}\left(x_{1}\right) & \cdots & H_{m}\left(x_{1}\right) \\
\vdots & \vdots & \ddots & \vdots \\
H_{1}\left(x_{P-1}\right) & H_{2}\left(x_{P-1}\right) & \cdots & H_{m}\left(x_{P-1}\right) \\
H_{1}\left(x_{P}\right) & H_{2}\left(x_{P}\right) & \cdots & H_{m}\left(x_{P}\right)
\end{array}\right)\left(\begin{array}{c}
\mathbf{V}_{1} \\
\mathbf{V}_{2} \\
\vdots \\
\mathbf{V}_{M}
\end{array}\right)^{T}\right]^{-1}\left(\begin{array}{c}
X_{0}\left(\xi_{0}\right) \\
X_{0}\left(\xi_{1}\right) \\
\vdots \\
X_{0}\left(\xi_{P}\right)
\end{array}\right)
$$

With this, we have now completed the process to build the approximation for the solution.

\subsubsection{Deterministic equation associated with the stochastic diffusion 6.1}

Set

$$
y(t, \xi)=\mathbb{E}\left[X_{t}(\xi)\right]
$$

then, $y(t, \xi)$ solves the differential equation

$$
\begin{aligned}
\frac{\partial y}{\partial t} & =\nu \frac{\partial^{2} y}{\partial \xi^{2}}+f \\
\left.y\right|_{t=0} & =\mathbb{E}\left(X_{0}\right) .
\end{aligned}
$$

We solve numerically this equation by using the Matlab library pdepe and we compare our results by using the spectral method with the one obtained with the pdepe Matlab library. 


\section{Results on the simulation}

We have the following graphs of simulations using this method with differents values of $J^{N, M}, N=7,8$. We make a comparison with the solution of the deterministic equation, as was described in subsection 6.2.1 by using the matlab library pdepe.

First we show the result on the simulation for the evaluation functional. The second group of graphs shows the simulation for the second functional. The results were obtained with the coefficient $\nu=0.1$.

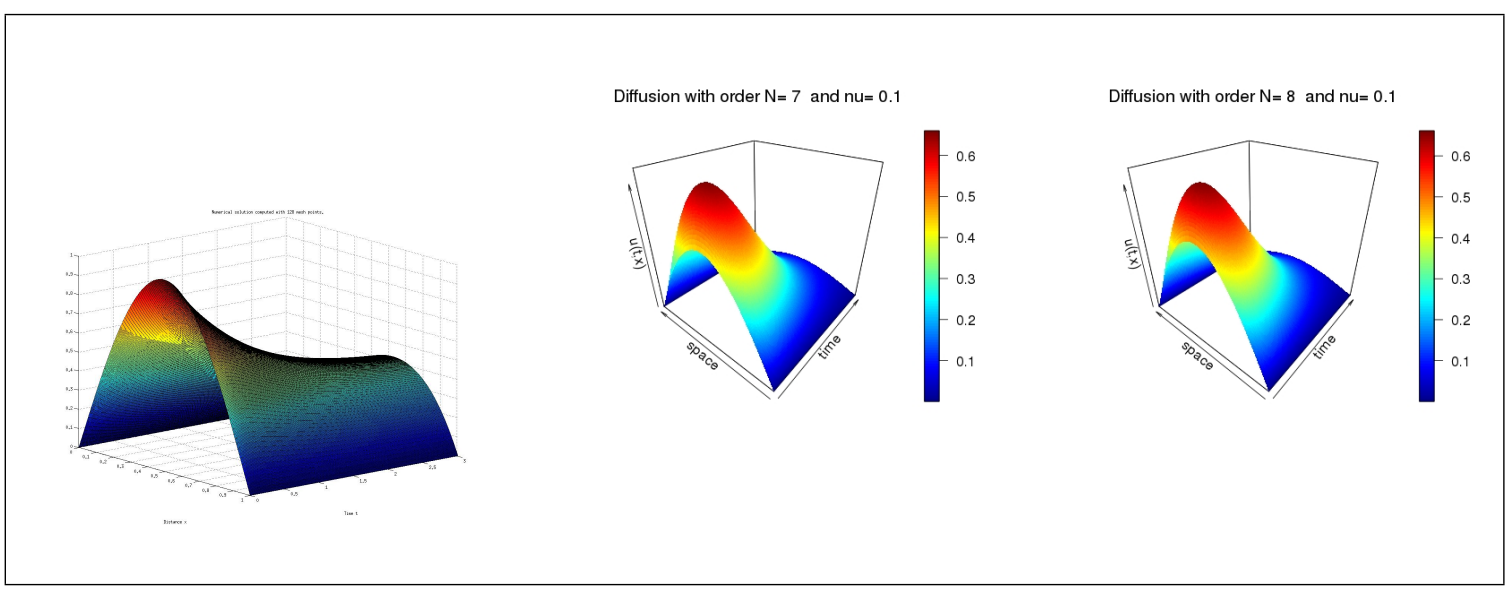

Figure 1: Simulations for the Diffusion equation with the spectral method, for $N=7,8$ and $\nu=0.1$ with $u_{0}^{\xi_{0}}(g)=g\left(\xi_{0}\right)$.

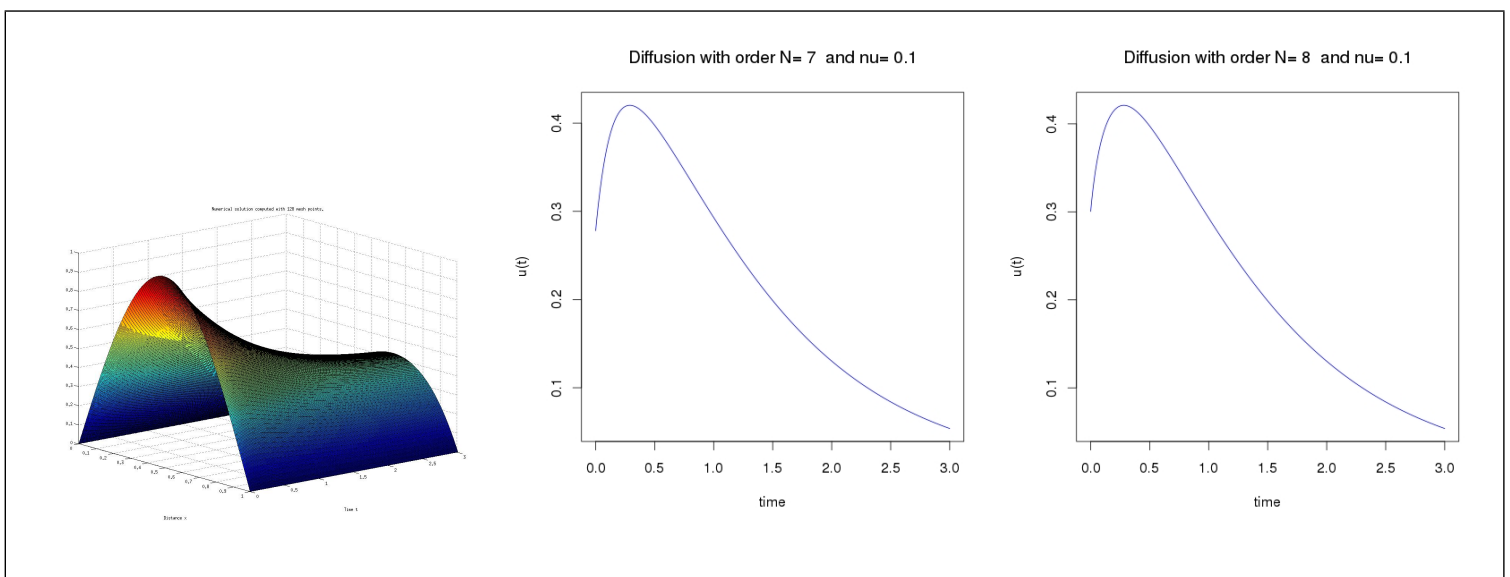

Figure 2: Simulations for the Diffusion equation with the spectral method, for $N=7,8, \nu=0.1$ and $\left.u_{0}(g)=\int_{0}^{1} g(\xi) d \xi\right)$..

\subsection{Stochastic Fisher-KPP Equation in an interval}

Set $\mathcal{H}=L^{2}(0,1)$. We consider the stochastic Fisher-KPP equation in the interval $[0,1]$ :

$$
\begin{aligned}
d X(t, \xi) & =\left[\nu \partial_{\xi}^{2} X(t, \xi)+X(t, \xi)(1-X(t, \xi))\right] d t+d W_{t}(t, \xi), \quad t>0, \quad \xi \in(0,1) \\
X(t, 0) & =X(t, 1)=0, \quad t>0 \\
X(0, \xi) & =x(\xi), \quad x \in \mathcal{H}
\end{aligned}
$$

$W$ is a cylindrical Wiener process on $\mathcal{H}$, associated to a stochastic basis $\left(\Omega, \mathcal{F}, \mathbb{P},\left(\mathcal{F}_{t}\right)_{t \geq 0}\right)$. $\nu$ is the viscosity coefficient. We will consider the initial condition $X(0, \xi)=\operatorname{sech}^{2}(5(\xi-0.5))$. 
We rewrite the Fisher-KPP equation as an abstract differential equation in $\mathcal{H}$. Set $A=\nu \partial_{\xi}^{2}$ and $B(x)=x(1-x), x \in \mathcal{H}$, with domains $D(A)=H^{2}(0,1) \cap H_{0}^{1}(0,1)$ and $D(B)=H_{0}^{1}(0,1)$, respectively. Then, (6.11) can be rewriten as

$$
\begin{aligned}
d X & =[A X+B(X)] d t+d W_{t} \\
X(0) & =x \quad x \in \mathcal{H} .
\end{aligned}
$$

The operator $A$ is selfadjoint with a complete orthonormal system of eigenfunctions in $\mathcal{H}$ given by

$$
e_{k}(\xi)=\sqrt{2} \sin (k \pi \xi), \quad \xi \in[0,1], k \in \mathbb{N} .
$$

Moreover $A$ satisfies $A e_{k}=-\nu \pi^{2} k^{2} e_{k}$, for $k \in \mathbb{N}$.

As before we define $u(t, x)=\mathbb{E}\left[u_{0}\left(X_{t}^{x}\right)\right]$ and then $u(t, x)$ satisfies the Kolmogorov equation

$$
\frac{\partial u}{\partial t}=\frac{1}{2} \operatorname{Tr}\left(Q D^{2} u\right)+\langle A x, D u\rangle_{\mathcal{H}}+\langle B(x), D u\rangle_{\mathcal{H}}, \quad x \in D(A) .
$$

Results on existence and uniqueness of the solution to the Kolmogorov equation can be found, for instance, in [7, Chapter 4].

About the functional $u_{0}: \mathcal{H} \rightarrow \mathbb{R}$ we will consider two cases :

$$
u_{0}^{\xi_{0}}(g):=g\left(\xi_{0}\right) . \quad \text { for fixed } \xi_{0} \in(0,1)
$$

and

$$
u_{0}(g):=\int_{0}^{1} g(\xi) d \xi
$$

We now apply the numerical method. We write the solution $u$ as

$$
u(t, x)=\sum_{\boldsymbol{n}} u_{\boldsymbol{n}}(t) H_{\boldsymbol{n}}(x)
$$

and by following the procedure done before we arrive to an infinite system of ordinary differential equations:

$$
\dot{u}_{\boldsymbol{m}}(t)=-u_{\boldsymbol{m}}(t) \lambda_{\boldsymbol{m}}+\sum_{\boldsymbol{n} \in \mathcal{J}} u_{\boldsymbol{n}}(t) C_{\boldsymbol{n}, \boldsymbol{m}}, \quad \boldsymbol{n}, \boldsymbol{m} \in \mathcal{J}
$$

where $C_{n, m}$ is given by

$$
C_{\boldsymbol{n}, \boldsymbol{m}}=\int_{\mathcal{H}}\left\langle B(x), D_{x} H_{\boldsymbol{n}}(x)\right\rangle_{\mathcal{H}} H_{\boldsymbol{m}}(x) \mu(d x)
$$

we need to calculate the value of the constants $C_{\boldsymbol{n}, \boldsymbol{m}}$, then we need to calculate expressions such as $B(x), D_{x} H_{\boldsymbol{n}}(x)$.

Focus on the term $B(x)=x(1-x)$. By writing $x=\sum_{k} \beta_{k} e_{k}$, with $\beta_{k}:=\left\langle x, e_{k}\right\rangle_{\mathcal{H}}$ we have

$$
B(x)=\left(\sum_{k} \beta_{k} e_{k}\right)\left(1-\sum_{k} \beta_{k} e_{k}\right)=\sum_{k} \beta_{k} e_{k}-\sum_{k} \sum_{l} \beta_{l} \beta_{k} e_{l} e_{k}
$$

For the expression $D_{x} H_{\boldsymbol{n}}(x)$ we have

$$
D_{x} H_{\boldsymbol{n}}(x)=\sum_{j=1}^{\infty} \prod_{\substack{i=1 \\ i \neq j}}^{\infty} P_{n_{i}}\left(\left\langle x, \Lambda^{-1 / 2} e_{i}\right\rangle_{\mathcal{H}}\right) P_{n_{j}}^{\prime}\left(\left\langle x, \Lambda^{-1 / 2} e_{j}\right\rangle_{\mathcal{H}}\right) \Lambda^{-1 / 2} e_{j}
$$

Setting $\Lambda=(-A)^{-1}$ and by recalling that $A e_{j}=-\nu \pi^{2} j^{2} e_{j}$ we have $\Lambda^{-1 / 2} e_{j}=\sqrt{2 \nu} \pi|j| e_{j}$, and by using the last expression we have,

$$
C_{\boldsymbol{n}, \boldsymbol{m}}=\int_{\mathcal{H}} H_{\boldsymbol{m}}(x) \mu(d x) \sum_{j=1}^{\infty} \prod_{\substack{i=1 \\ i \neq j}}^{\infty} P_{n_{i}}\left(\left\langle x, \Lambda^{-1 / 2} e_{i}\right\rangle_{\mathcal{H}}\right) P_{n_{j}}^{\prime}\left(\left\langle x, \Lambda^{-1 / 2} e_{j}\right\rangle_{\mathcal{H}}\right) \sqrt{2 \nu} \pi|j|
$$




$$
\begin{aligned}
& \times {\left[\sum_{k} \beta_{k}\left\langle e_{k}, e_{j}\right\rangle_{\mathcal{H}}-\sum_{l} \sum_{k} \beta_{l} \beta_{k}\left\langle e_{l} e_{k}, e_{j}\right\rangle_{\mathcal{H}}\right] } \\
&=\int_{\mathcal{H}} \mu(d x) \sum_{j=1}^{\infty} \sqrt{2 \nu} \pi|j| P_{m_{j}}\left(\left\langle x, \Lambda^{-1 / 2} e_{j}\right\rangle_{\mathcal{H}}\right) P_{n_{j}}^{\prime}\left(\left\langle x, \Lambda^{-1 / 2} e_{j}\right\rangle_{\mathcal{H}}\right) \\
& \quad \times \prod_{\substack{i=1 \\
i \neq j}}^{\infty} P_{n_{i}}\left(\left\langle x, \Lambda^{-1 / 2} e_{i}\right\rangle_{\mathcal{H}}\right) P_{m_{i}}\left(\left\langle x, \Lambda^{-1 / 2} e_{i}\right\rangle_{\mathcal{H}}\right)\left[\beta_{j}-\sum_{l} \sum_{k} \beta_{l} \beta_{k}\left\langle e_{l} e_{k}, e_{j}\right\rangle_{\mathcal{H}}\right] .
\end{aligned}
$$

For $N_{1} \in \mathbb{N}$ define as before the set $S_{N_{1}}=\left\{\boldsymbol{n}_{1}, \boldsymbol{n}_{2}, \ldots, \boldsymbol{n}_{N_{1}}: \boldsymbol{n}_{i} \in J^{M, N}, i=1, \ldots, N_{1}\right\}$. Moreover, for $\boldsymbol{n}, \boldsymbol{m} \in S_{M}$ define

$$
\begin{aligned}
\bar{C}_{\boldsymbol{n}, \boldsymbol{m}}:=\sum_{j=1}^{M} \int_{\mathbb{R}^{M}} P_{m_{j}}\left(\xi_{j}\right) P_{n_{j}}^{\prime}\left(\xi_{j}\right) \mu\left(d \xi_{j}\right) \\
\quad \times \prod_{\substack{i=1 \\
i \neq j}}^{M} P_{m_{i}}\left(\xi_{i}\right) P_{n_{i}}\left(\xi_{i}\right) \frac{\mu\left(d \xi_{i}\right)}{\lambda_{i}}\left[\beta_{j}-\sum_{l=1}^{M} \sum_{k=1}^{M} \beta_{l} \beta_{k}\left\langle e_{l} e_{k}, e_{j}\right\rangle_{\mathcal{H}}\right] .
\end{aligned}
$$

and the finite system of ordinary differential equations:

$$
\dot{u}_{\boldsymbol{m}}(t)=-u_{\boldsymbol{m}}(t) \lambda_{\boldsymbol{m}}+\sum_{\boldsymbol{n} \in S_{M}} u_{\boldsymbol{n}}(t) \bar{C}_{\boldsymbol{n}, \boldsymbol{m}}, \quad \text { for each } \boldsymbol{m} \in S_{M} \text { and } \boldsymbol{n} \in S_{M} .
$$

Then (6.15) approximates to the infinite system of ordinary differential equations (6.13) when $N, M \rightarrow$ $\infty$. We use the system (6.15) to approximate the solution of the FPK equation associated to the Fisher-KPP equation.

\subsubsection{Deterministic equation associated with the stochastic Fisher-KPP Equation.}

Set

$$
y(t, \xi)=\mathbb{E}\left[X_{t}(\xi)\right]
$$

then, $y(t, \xi)$ solves the differential equation

$$
\begin{aligned}
\frac{\partial y}{\partial t} & =\nu \frac{\partial^{2} y}{\partial \xi^{2}}++y(t, \xi)[1-y(t, \xi)] \\
\left.y\right|_{t=0} & =\mathbb{E}\left(X_{0}\right) .
\end{aligned}
$$

We solve numerically this equation by using the Matlab library pdepe and we compare our results by using the spectral method with the one obtained with the pdepe Matlab library.

\section{Results on the simulation}

We have the following graphs of simulations using the proposed method with differents values of $J^{N, M}$, $N=4,5$. We make a comparison with the solution of the deterministic equation, as was described in subsection 6.3.1. by using the matlab library pdepe.

We show the results on the simulation for the evaluation functional. The second graph shows the 
simulation for the second functional. The results were obtained with the coefficient $\nu=0.1$.

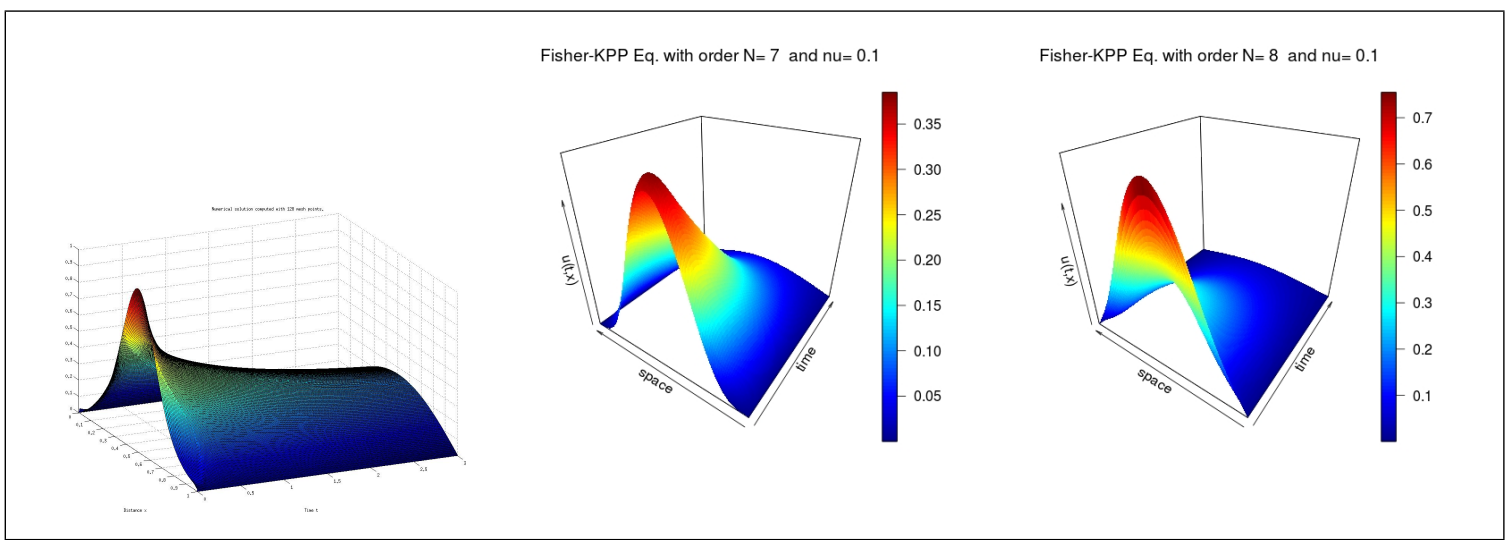

Figure 3: Simulations for the Fisher-KPP equation with the Matlab library pdepe and with the spectral method for $N=7,8, u_{0}^{\xi_{0}}(g)=g\left(\xi_{0}\right)$.

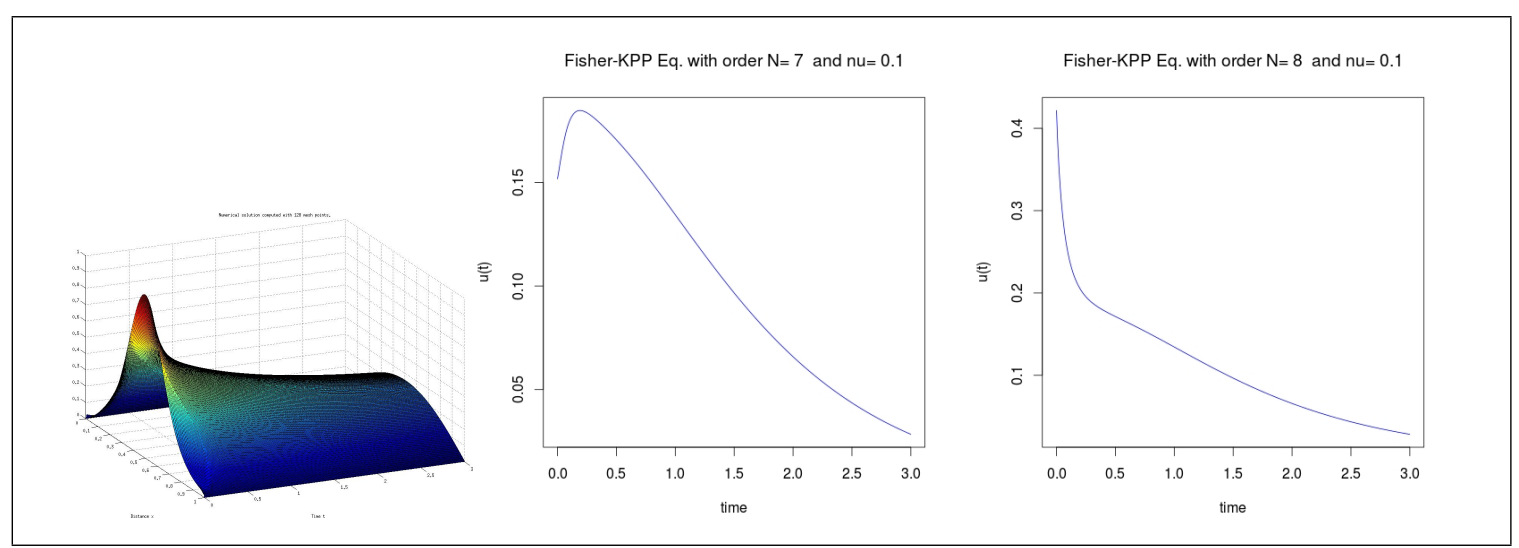

Figure 4: Simulations for the Fisher-KPP equation with the Matlab library pdepe and with the spectral method for $N=7,8, u_{0}(g)=\int_{0}^{1} g(\xi) d \xi$.

\subsection{Stochastic Burgers Equation in an interval}

Set $\mathcal{H}=L^{2}(0,1)$. We consider the stochastic Burgers equation in the interval $[0,1]$ :

$$
\begin{aligned}
d X(t, \xi) & =\left[\nu \partial_{\xi}^{2} X(t, \xi)+\frac{1}{2} \partial_{\xi}\left(X^{2}(t, \xi)\right)\right] d t+d W_{t}(t, \xi), \quad t>0, \quad \xi \in(0,1) \\
X(t, 0) & =X(t, 1)=0, \quad t>0 \\
X(0, \xi) & =x(\xi), \quad x \in \mathcal{H}
\end{aligned}
$$

$W$ is a cylindrical Wiener process on $\mathcal{H}$, associated to a stochastic basis $\left(\Omega, \mathcal{F}, \mathbb{P},\left(\mathcal{F}_{t}\right)_{t \geq 0}\right) . \nu$ is the viscosity coefficient.

We rewrite the Burgers equation as an abstract differential equation in $\mathcal{H}$. Set $A=\nu \partial_{\xi}^{2}$ and $B(x)=$ $\frac{1}{2} \partial_{\xi}\left(x^{2}\right), x \in \mathcal{H}$, with domains $D(A)=H^{2}(0,1) \cap H_{0}^{1}(0,1)$ and $D(B)=H_{0}^{1}(0,1)$, respectively. Then, (6.17) can be rewriten as

$$
\begin{aligned}
d X & =[A X+B(X)] d t+d W_{t} \\
X(0) & =x \quad x \in \mathcal{H} .
\end{aligned}
$$

The operator $A$ is selfadjoint with a complete orthonormal system of eigenfunctions in $\mathcal{H}$ given by

$$
e_{k}(\xi)=\sqrt{2} \sin (k \pi \xi), \quad \xi \in[0,1], k \in \mathbb{N} .
$$


Moreover $A$ satisfies $A e_{k}=-\nu \pi^{2} k^{2} e_{k}$, for $k \in \mathbb{N}$.

As before we define $u(t, x)=\mathbb{E}\left[u_{0}\left(X_{t}^{x}\right)\right]$ and then $u(t, x)$ satisfies the Kolmogorov equation

$$
\frac{\partial u}{\partial t}=\frac{1}{2} \operatorname{Tr}\left(Q D^{2} u\right)+\langle A x, D u\rangle_{\mathcal{H}}+\langle B(x), D u\rangle_{\mathcal{H}}, \quad x \in D(A) .
$$

Results on existence and uniqueness of the solution to the Kolmogorov equation can be found, for instance, in [7. Chapter 5].

We will consider again two types of functionals :

and

$$
u_{0}^{\xi_{0}}(g):=g\left(\xi_{0}\right) . \quad \text { for fixed } \xi_{0} \in(0,1)
$$

$$
u_{0}(g):=\int_{0}^{1} g(\xi) d \xi
$$

We now apply the numerical method. We write the solution $u$ as

$$
u(t, x)=\sum_{n} u_{\boldsymbol{n}}(t) H_{\boldsymbol{n}}(x)
$$

and by following the procedure done before we arrive to an infinite system of ordinary differential equations:

$$
\dot{u}_{\boldsymbol{m}}(t)=-u_{\boldsymbol{m}}(t) \lambda_{\boldsymbol{m}}+\sum_{\boldsymbol{n} \in \mathcal{J}} u_{\boldsymbol{n}}(t) C_{\boldsymbol{n}, \boldsymbol{m}}, \quad \boldsymbol{n}, \boldsymbol{m} \in \mathcal{J}
$$

where $C_{\boldsymbol{n}, \boldsymbol{m}}$ is given by

$$
C_{\boldsymbol{n}, \boldsymbol{m}}=\int_{\mathcal{H}}\left\langle B(x), D_{x} H_{\boldsymbol{n}}(x)\right\rangle_{\mathcal{H}} H_{\boldsymbol{m}}(x) \mu(d x)
$$

we need to calculate the value of the constants $C_{n, m}$, then we need to calculate expressions such as $B(x), D_{x} H_{\boldsymbol{n}}(x)$.

Focus on the term $B(x)=\frac{1}{2} \partial_{\xi}\left(x^{2}\right)$. By writing $x=\sum_{k} \beta_{k} e_{k}$, with $\beta_{k}:=\left\langle x, e_{k}\right\rangle_{\mathcal{H}}$ we have

$$
B(x)=\frac{1}{2} \partial_{\xi}\left(\sum_{k} \beta_{k} e_{k}\right)^{2}=\frac{1}{2} \partial_{\xi}\left[\sum_{l} \sum_{k} \beta_{l} \beta_{k} e_{l} e_{k}\right]=\frac{1}{2} \sum_{l} \sum_{k} \beta_{l} \beta_{k}\left(e_{l} e_{k}^{\prime}+e_{l}^{\prime} e_{k}\right) .
$$

For the expression $D_{x} H_{\boldsymbol{n}}(x)$ we have

$$
D_{x} H_{\boldsymbol{n}}(x)=\sum_{j=1}^{\infty} \prod_{\substack{i=1 \\ i \neq j}}^{\infty} P_{n_{i}}\left(\left\langle x, \Lambda^{-1 / 2} e_{i}\right\rangle_{\mathcal{H}}\right) P_{n_{j}}^{\prime}\left(\left\langle x, \Lambda^{-1 / 2} e_{j}\right\rangle_{\mathcal{H}}\right) \Lambda^{-1 / 2} e_{j}
$$

Setting $\Lambda=(-A)^{-1}$ and by recalling that $A e_{j}=-\nu \pi^{2} j^{2} e_{j}$ we have $\Lambda^{-1 / 2} e_{j}=\sqrt{2 \nu} \pi|j| e_{j}$, and by using the last expression we have,

$$
\begin{aligned}
C_{\boldsymbol{n}, \boldsymbol{m}}= & \frac{1}{2} \int_{\mathcal{H}} H_{\boldsymbol{m}}(x) \mu(d x) \sum_{j=1}^{\infty} \prod_{\substack{i=1 \\
i \neq j}}^{\infty} P_{n_{i}}\left(\left\langle x, \Lambda^{-1 / 2} e_{i}\right\rangle_{\mathcal{H}}\right) P_{n_{j}}^{\prime}\left(\left\langle x, \Lambda^{-1 / 2} e_{j}\right\rangle_{\mathcal{H}}\right) \sqrt{2 \nu} \pi|j| \\
& \times \sum_{l} \sum_{k} \beta_{l} \beta_{k}\left\langle e_{l} e_{k}^{\prime}+e_{l}^{\prime} e_{k}, e_{j}\right\rangle_{\mathcal{H}} \\
= & \frac{1}{2} \int_{\mathcal{H}} \mu(d x) \sum_{j=1}^{\infty} \sqrt{2 \nu} \pi|j| P_{m_{j}}\left(\left\langle x, \Lambda^{-1 / 2} e_{j}\right\rangle_{\mathcal{H}}\right) P_{n_{j}}^{\prime}\left(\left\langle x, \Lambda^{-1 / 2} e_{j}\right\rangle_{\mathcal{H}}\right) \\
& \times \prod_{\substack{i=1 \\
i \neq j}}^{\infty} P_{n_{i}}\left(\left\langle x, \Lambda^{-1 / 2} e_{i}\right\rangle_{\mathcal{H}}\right) P_{m_{i}}\left(\left\langle x, \Lambda^{-1 / 2} e_{i}\right\rangle_{\mathcal{H}}\right) \sum_{l} \sum_{k} \beta_{l} \beta_{k}\left\langle e_{l} e_{k}^{\prime}+e_{l}^{\prime} e_{k}, e_{j}\right\rangle_{\mathcal{H}}
\end{aligned}
$$


For $N_{1} \in \mathbb{N}$ define as before the set $S_{N_{1}}=\left\{\boldsymbol{n}_{1}, \boldsymbol{n}_{2}, \ldots, \boldsymbol{n}_{N_{1}}: \boldsymbol{n}_{i} \in J^{M, N}, i=1, \ldots, N_{1}\right\}$. Moreover, for $\boldsymbol{n}, \boldsymbol{m} \in S_{M}$ define

$$
\begin{aligned}
\bar{C}_{\boldsymbol{n}, \boldsymbol{m}}:=\frac{1}{2} \sum_{j=1}^{M} & \sqrt{2 \nu} \pi|j| \int_{\mathbb{R}^{M}} P_{m_{j}}\left(\xi_{j}\right) P_{n_{j}}^{\prime}\left(\xi_{j}\right) \mu\left(d \xi_{j}\right) \\
& \times \prod_{\substack{i=1 \\
i \neq j}}^{M} P_{m_{i}}\left(\xi_{i}\right) P_{n_{i}}\left(\xi_{i}\right) \mu\left(d \xi_{i}\right) \sum_{l=1}^{M} \sum_{k=1}^{M} \beta_{l} \beta_{k}\left\langle e_{l} e_{k}^{\prime}+e_{l}^{\prime} e_{k}, e_{j}\right\rangle_{\mathcal{H}}
\end{aligned}
$$

and the finite system of ordinary differential equations:

$$
\dot{u}_{\boldsymbol{m}}(t)=-u_{\boldsymbol{m}}(t) \lambda_{\boldsymbol{m}}+\sum_{\boldsymbol{n} \in S_{M}} u_{\boldsymbol{n}}(t) \bar{C}_{\boldsymbol{n}, \boldsymbol{m}}, \quad \text { for each } \boldsymbol{m} \in S_{M} \text { and } \boldsymbol{n} \in S_{M} .
$$

Then (6.22) approximates to the infinite system of ordinary differential equations (6.20) when $N, M \rightarrow$ $\infty$. We use the system (6.22) to approximate the solution of the FPK equation associated with the Burgers equation.

\subsubsection{Deterministic equation associated with the stochastic Burgers Equation.}

Set

$$
y(t, \xi)=\mathbb{E}\left[X_{t}(\xi)\right]
$$

then, $y(t, \xi)$ solves the differential equation

$$
\begin{aligned}
\frac{\partial y}{\partial t} & =\nu \frac{\partial^{2} y}{\partial \xi^{2}}++\frac{1}{2} \partial_{\xi}\left(y^{2}(t, \xi)\right) \\
\left.y\right|_{t=0} & =\mathbb{E}\left(X_{0}\right) .
\end{aligned}
$$

We solve numerically this equation by using the Matlab library pdepe and we compare our results by using the spectral method with the one obtained with the pdepe Matlab library.

\section{Results on the simulation}

The following graphs show simulations by using the proposed method with differents values of $J^{N, M}$, $N=4,5$. We make a comparison with the solution of the deterministic equation, as was described in subsection 6.4.1. by using the matlab library pdepe.

Tthe results on the simulation for the evaluation functional are in the first group of graphs. The second graph shows the simulation for the second functional. The results were obtained with the coefficient $\nu=0.2,0.1,0.01$.

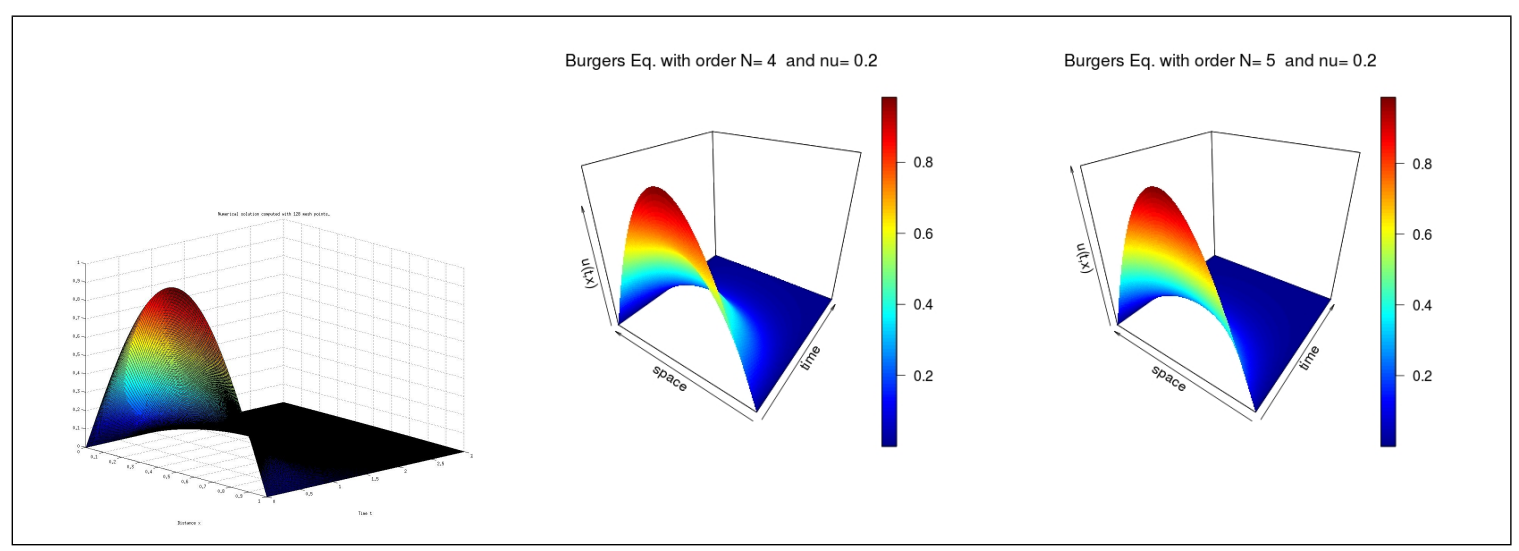

Figure 5: Simulations for the Burgers equation with the Matlab library pdepe and with the spectral method for $N=4,5, u_{0}^{\xi_{0}}(g)=g\left(\xi_{0}\right)$. 


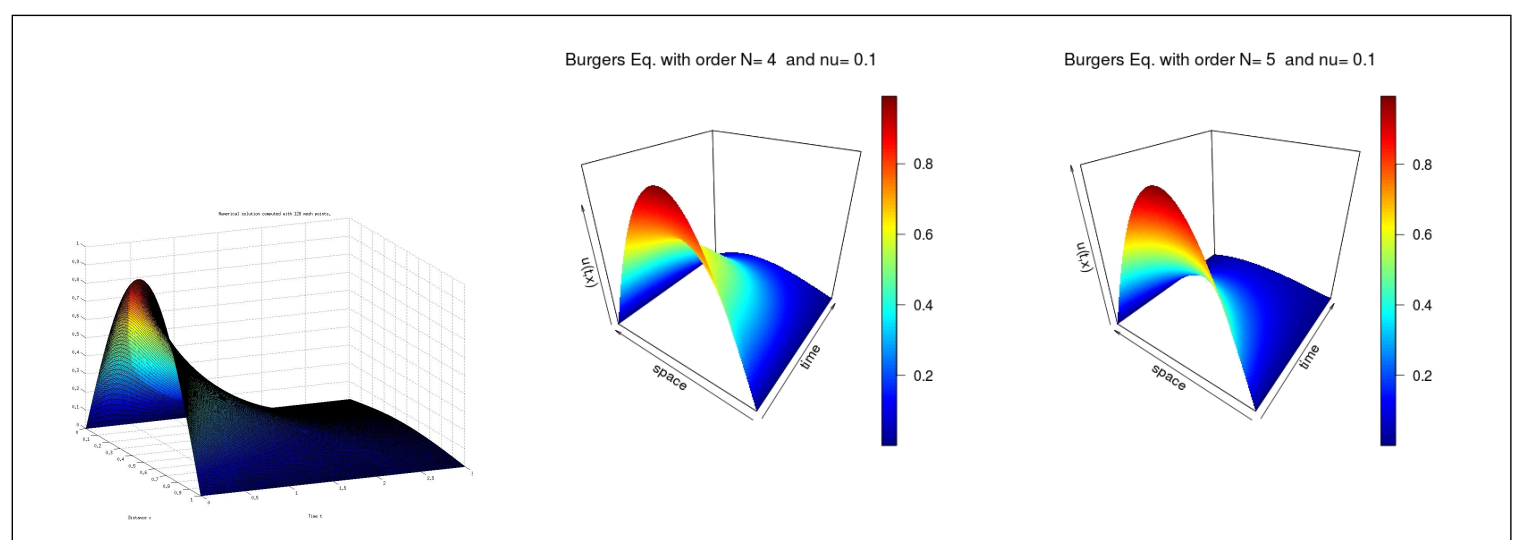

Figure 6: Simulations for the Burgers equation with the Matlab library pdepe and with the spectral method for $N=4,5, u_{0}^{\xi_{0}}(g)=g\left(\xi_{0}\right)$.

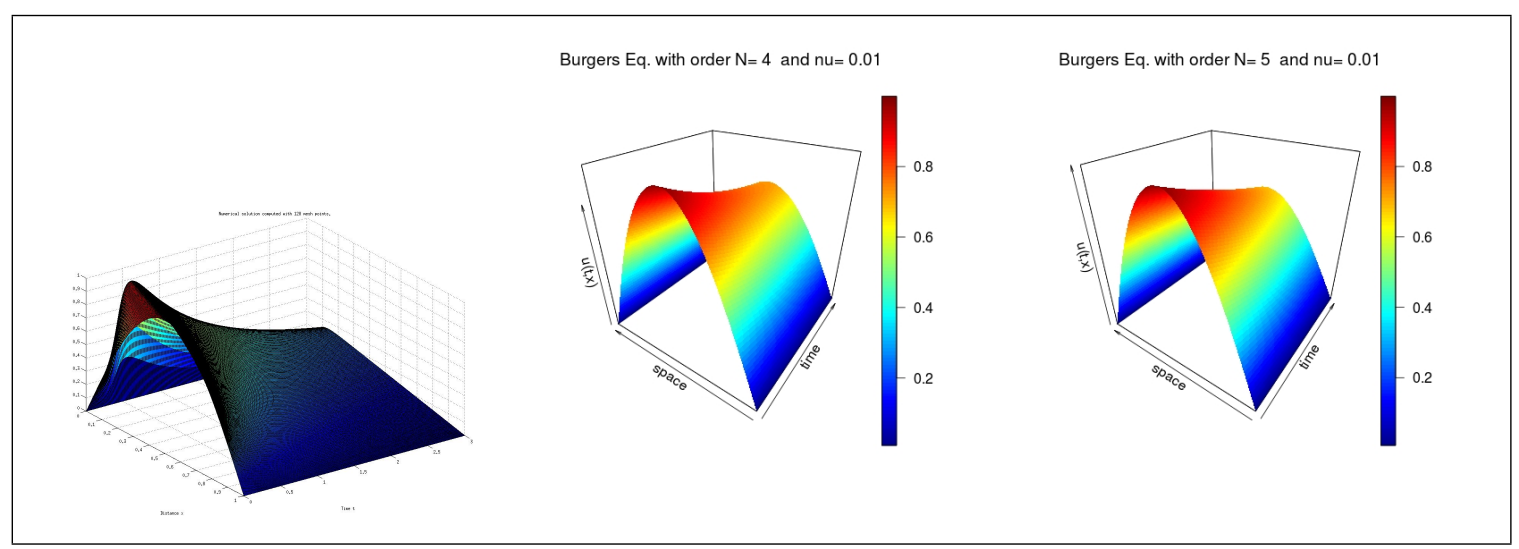

Figure 7: Simulations for the Burgers equation with the Matlab library pdepe and with the spectral method for $N=4,5, u_{0}^{\xi_{0}}(g)=g\left(\xi_{0}\right)$.

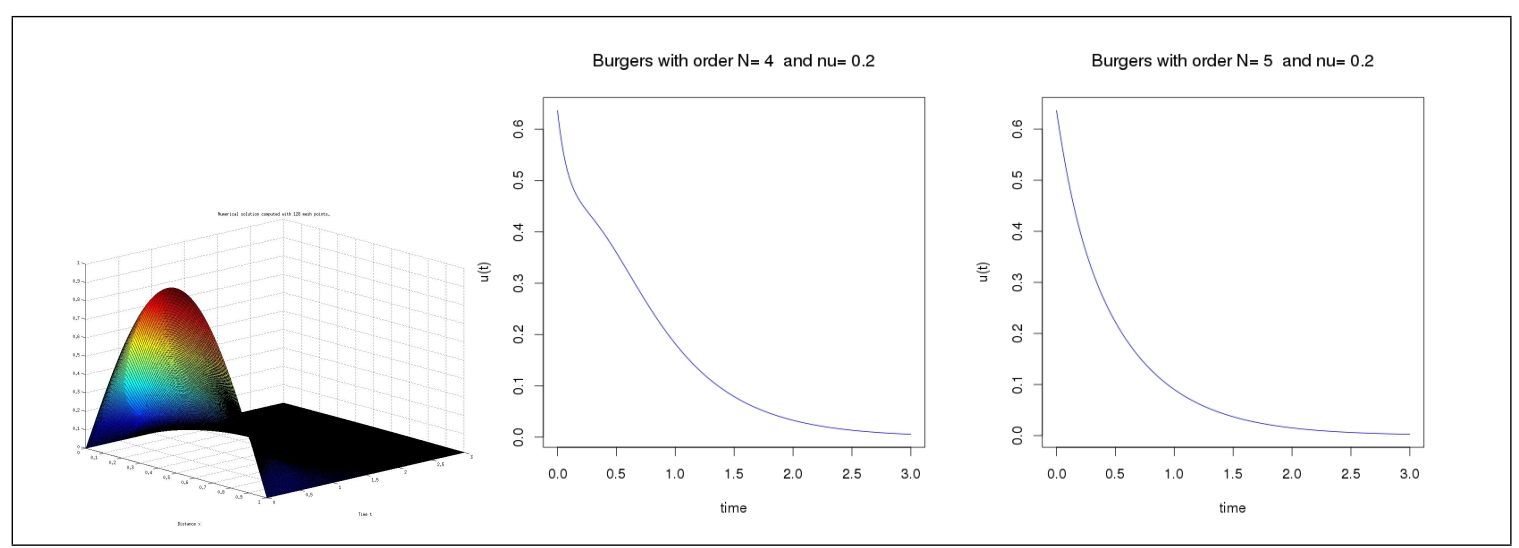

Figure 8: Simulations for the Burgers equation with the Matlab library pdepe and with the spectral method for $N=4,5, u_{0}(g)=\int_{0}^{1} g(\xi) d \xi$. 


\section{CONCLUSIONS.}

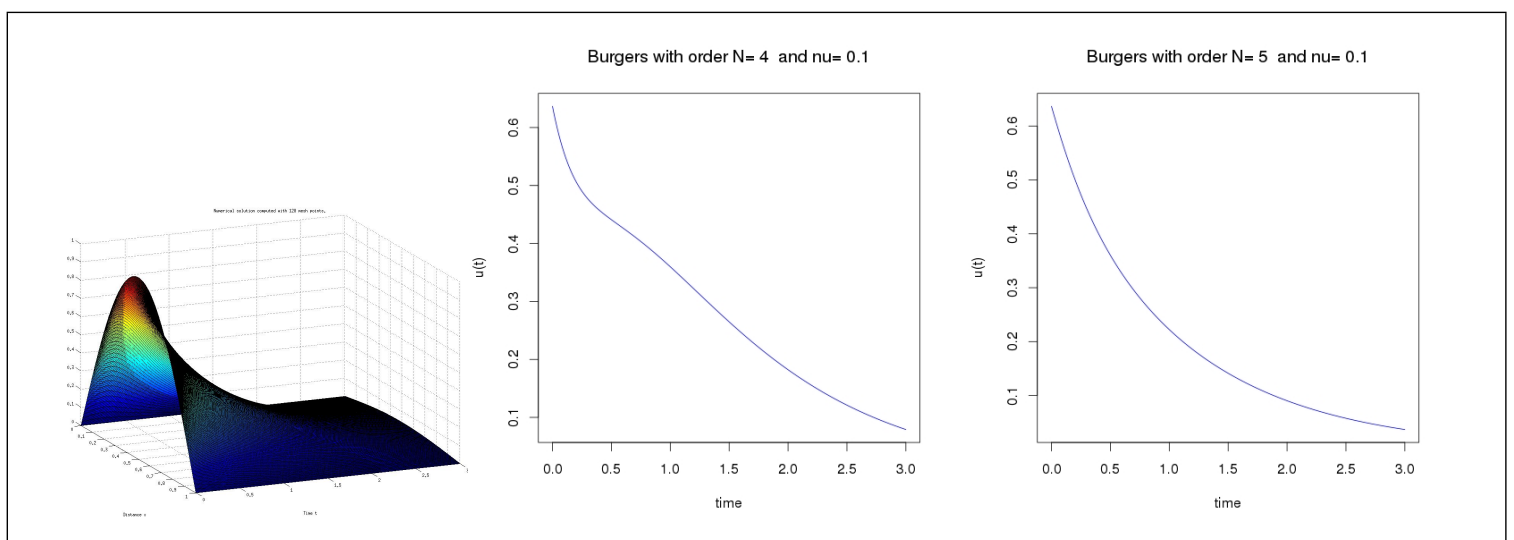

Figure 9: Simulations for the Burgers equation with the Matlab library pdepe and with the spectral method for $N=4,5, u_{0}(g)=\int_{0}^{1} g(\xi) d \xi$.
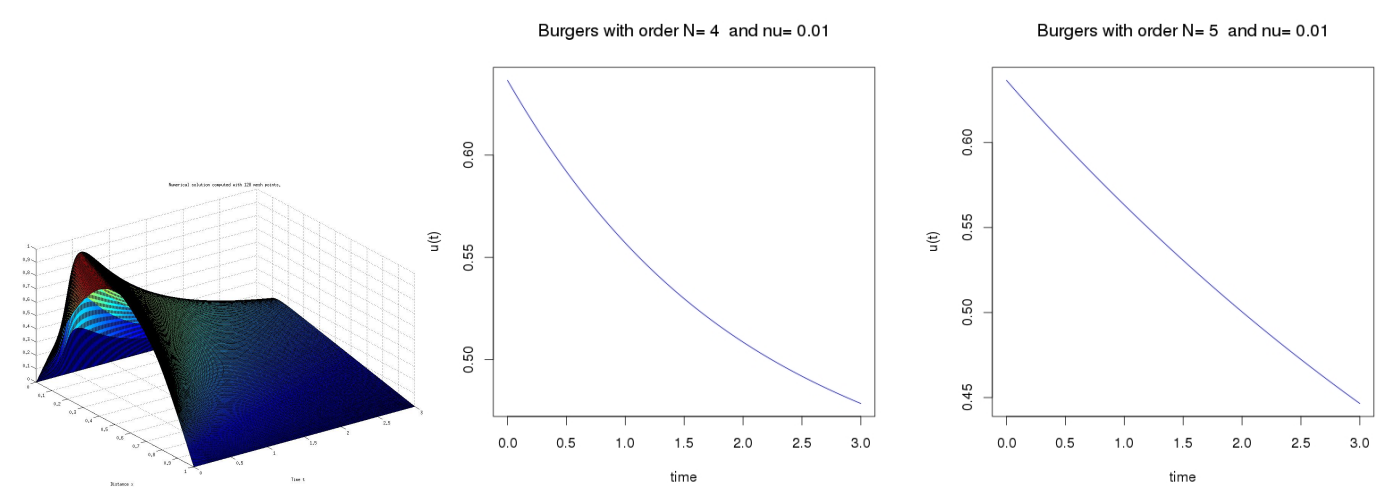

Figure 10: Simulations for the Burgers equation with the Matlab library pdepe and with the spectral method for $N=4,5, u_{0}(g)=\int_{0}^{1} g(\xi) d \xi$.

\section{Conclusions.}

In this paper we introduced a numerical method to solve Fokker-Plank-Kolmogorov equations and we tested this method by applying it to the Kolmogorov equations associated to three stochastic partial differential equations: a stochastic diffusion, a Fisher-KPP stochastic equation and a stochastic Burgers equation in $1 D$, in a simple domain in the three cases. The results obtained are really promising. However, there are a few limitations. The first is that the noise in the SPDE is restricted to the additive case and to cover the multiplicative case seems unfeasible at this moment. Indeed, even if one is able to prove existence and uniqueness of an invariant measure $\nu$ for the Ornstein-Uhlenbeck semigroup associated with the SPDE, there would remain the fully characterize of the measure and to find a basis for the Hilbert Space $L^{2}(\mathcal{H}, \nu)$. Another issue is that we have applied the method to very simple domains, However, to cover the cases with complex domains one can use ideas of domain decomposition techniques similar to those used in spectral element methods. This is part of a forthcoming paper.

The method can be adapted to cover the Fokker-Plank equations associated with SPDE's, this will be studied in a subsequent work.

Acknowledgement. The research leading to these results has received funding from the People Programme (Marie Curie Actions) of the European Union's Seventh Framework Programme (FP7/20072013) under the project NEMOH, REA grant agreement $n$. 289976. 


\section{References}

[1] V. Barbu, G. Da Prato: The Kolmogorov equation for a 2D Navier-Stokes stochastic flow in a channel. nonlinear Analysis. 69, pp. 940-949. (2008).

[2] V. Bogachev, G. Da Prato, M. Röeckner: Existence results for Fokker-Planck equations in Hilbert space. Seminar on Stochastic Analysis, Random Fields and Applications VI. Progress in Probability Volume 63, 2011, pp 23-35 (2010)

[3] Cameron R.H., Martin W. T.: The orthogonal development of non-linear functionals in series of Fourier-Hermite functionals. Annals of Mathematics 48 (2): 385-392. (1947).

[4] P.-L. Chow: Infinte-dimensional Kolmogorov equations in Gauss-Sobolev spaces;. Stoch. Analy. Applic. 14, 257-282. (1996).

[5] P.-L. Chow: Infinite-dimensional parabolic equations in Gauss-Sobolev spaces. Comm. Stoch. Analy, 1, 71-86. (2007).

[6] P.-L. chow: Stochastic partial differential equations. Chapman and Hall/CRC. (2007)

[7] G. Da Prato: Kolmogorov equations for stochastic partial differential equations. Advanced Courses in Mathematics - CRM Barcelona. Birkhäuser. (2004).

[8] G. Da Prato, A. Debussche : $m$-Dissipativity of Kolmogorov Operators Corresponding to Burgers Equations with Space-time White Noise. Potential Analysis. Volume 26, Issue 1, pp 31-55. (2007).

[9] G. Da Prato, J. Zabczyk : Second order partial differential equations in Hilbert spaces. Cambridge University Press, (2002).

[10] G. Da Prato, J. Zabczyk: Stochastic equations in infinite dimensions. Cambridge University Press, (1992).

[11] G. Da Prato, F. Flandoli, M. Röckner: Fokker-Planck Equations for SPDE with Non-trace-class Noise. Communications in Mathematics and Statistics. Volume 1, Issue 3, pp 281-304, (2013).

[12] Doostan A., laccarino G.: "A least-squares approximation of partial differential equations with high dimensional random inputs" Journal of Computational Physics, Vol. 228, No. 12, pp. 43324345, 2009.

[13] M. Giles: Improved multilevel Monte Carlo convergence using the Milstein scheme, Preprints NA-06/22, Oxford University Computing Laboratory, Parks Road, Oxford, U.K., (2006).

[14] M. B. Giles: Multilevel Monte Carlo path simulation, Oper. Res., 56 (2008), pp. 607-617.

[15] A.J. Goldberg, J.L. Schwartz: Systems of Ordinary Differential Equations : An Introduction. Published by Joanna Cotler Books. (1972).

[16] S. Heinrich : Multilevel Monte Carlo Methods Large Scale Scientific Computing, Third International Conference, LSSC 2001, Sozopol, Bulgaria, June 6-10, (2001), vol. 2179 of Lecture Notes in Computer Science, Springer, pp. 58-67 (2001).

[17] T. Y. Hou, W. Luo, B. Rozovskii, and H.M. Zhou:Wiener Chaos Expansions and Numerical Solutions of Randomly Forced Equations of Fluid Mechanics, J. Comput. Phys. , 216 , 687-706 (2006).

[18] T. Y. Hou , W. Luo, B. Rozovskii , H.M. Zhou: Wiener chaos expansions and numerical solutions of randomly forced equations of fluid mechanics Journal of Computational Physics archive. Vol 216 Issue 2, (2006)

[19] P. Imkeller: Malliavin's calculus and applications in stochastic control and finance IMPAN Lecture Notes, Vol. 1, Warsaw (2008). 
[20] A. Jentzen, P.E. Kloeden: Taylor approximations for stochastic partial differential equations. CBMS-NSF Regional Conference Series in Applied Mathematics, 83. Society for Industrial and Applied Mathematics (SIAM), Philadelphia, PA, (2011).

[21] P. E. Kloeden, E. Platen: Numerical Solution of Stochastic Differential Equations. Stochastic Modelling and Applied Probability, Vol. 23, Springer (1992).

[22] O. P. Le Matre, O.M. Knio: Spectral methods for uncertainty quantification. With applications to computational fluid dynamics. Scientific Computation. Springer, New York, (2010).

[23] J. L. Lions: Quelques methodes de resolution des problemes aux limites non lineaires, Dunod, Paris (1969).

[24] S. V. Lototsky. Chaos Approach to Nonlinear Filtering. In: D. Crisan and B. L. Rozovskii (editors), The Oxford Handbook of Nonlinear Filtering, pp. 231-264, Oxford University Press, (2011).

[25] S. V. Lototsky and B. L. Rozovskii. Wiener Chaos Solutions of Linear Stochastic Evolution Equations. Annals of Probability, Vol. 34, No. 2, pp. 638-662, (2006)

[26] J. Ming, M. Gunzburger: Efficient numerical methods for stochastic partial differential equations through transformation to equations driven by correlated noise. Int. J. Uncertain. Quantif. 3, no. 4, 321-339, (2013).

[27] W. Luo, Wiener Chaos expansion and numerical solutions for stochastic partial differential equations. PhD thesis. Instituto de Tecnologa de California (2006).

[28] E. Platen: An introduction to numerical methods for stochastic differential equations. Acta numerica 8, 197-246, (1999).

[29] C. Schwab, E. Süli: Adaptive Galerkin approximation algorithms for Kolmogorov equations in infinite dimensions Stochastic Partial Differential Equations: Analysis and Computations. 1:204239. (2013). 